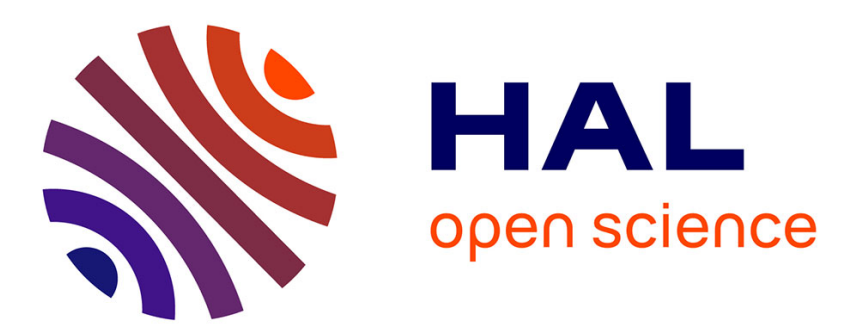

\title{
Les réemplois en architecture : étude de cas à Vaison-la-Romaine (Vasio Vocontiorum)
}

Caroline Lefebvre

\section{To cite this version:}

Caroline Lefebvre. Les réemplois en architecture: étude de cas à Vaison-la-Romaine (Vasio Vocontiorum). Mélanges de l'École française de Rome - Moyen Âge, 2017, Mélanges de l'Ecole française de Rome - Moyen Age, 129 (1), pp.177-193. 10.4000/mefrm.3570 . halshs-02425787

\section{HAL Id: halshs-02425787 https://shs.hal.science/halshs-02425787}

Submitted on 31 Dec 2019

HAL is a multi-disciplinary open access archive for the deposit and dissemination of scientific research documents, whether they are published or not. The documents may come from teaching and research institutions in France or abroad, or from public or private research centers.
L'archive ouverte pluridisciplinaire $\mathbf{H A L}$, est destinée au dépôt et à la diffusion de documents scientifiques de niveau recherche, publiés ou non, émanant des établissements d'enseignement et de recherche français ou étrangers, des laboratoires publics ou privés. 


\section{Mélanges de l'École française de Rome - Moyen Âge}

129-1 | 2017

Pise de la peste noire à la conquête florentine

(1348-1406). Nouvelles orientations pour l'histoire

d'une société en crise - Varia - Atelier doctoral : les réemplois en architecture, entre Antiquité et Moyen Âge

\section{Les réemplois en architecture : étude de cas à Vaison-la-Romaine (Vasio Vocontiorum)}

Caroline Lefebvre

\section{(2) OpenEdition}

Édition électronique

URL : http://journals.openedition.org/mefrm/3570

DOI : $10.4000 /$ mefrm. 3570

ISSN : $1724-2150$

Éditeur

École française de Rome

Édition imprimée

ISBN : 978-2-7283-1299-3

ISSN : 1123-9883

Ce document vous est offert par Bibliothèque Diderot de Lyon ENS

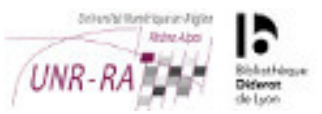

Référence électronique

Caroline Lefebvre, «Les réemplois en architecture : étude de cas à Vaison-la-Romaine (Vasio Vocontiorum) », Mélanges de l'École française de Rome - Moyen Âge [En ligne], 129-1 | 2017, mis en ligne le 28 septembre 2017, consulté le 23 juillet 2018. URL : http://journals.openedition.org/mefrm/3570 ; DOI : 10.4000/mefrm.3570 


\title{
Les réemplois en architecture Étude de cas à Vaison-la-Romaine (Vasio Vocontiorum)
}

\author{
Caroline LEFEBVRE
}

C. Lefebvre, Laboratoire IRAA (USR 3155) - Université Lumière Lyon 2, caro.lefebvre@hotmail.fr

La ville de Vaison-la-Romaine offre une étude à la fois riche et diversifiée lorsque l'on s'interroge sur le réemploi en architecture. À la suite de la conquête romaine qui prend fin au cours de la première moitié du $\mathrm{I}^{\mathrm{er}}$ siècle avant notre ère, la ville est implantée sur la rive droite d'un cours d'eau appelé Ouvèze. Elle est abandonnée au Moyen-Âge par la population qui migre sur la rive gauche où se développe un bourg médiéval. À l'époque moderne, la ville s'établie de nouveau sur la rive droite, là où avait été fondée la ville romaine, et s'y installe définitivement. Les diverses constructions qui se sont succédées de part et d'autre de l'Ouvèze, au cours des différentes époques, ont conservé des éléments réemployés qui constituent les précieux témoins de cette pratique. Les trois cas d'étude qui sont abordés se différencient par leur nature et leur chronologie mais ils attestent de l'usage d'une même technique pour leur mise en œeuvre. Ce phénomène du réemploi invite à s'interroger sur son processus et ses raisons, ainsi que sur les éléments réemployés, leur nouvelle destination et leur fonction, mais également leur provenance.

Vaison-la-Romaine, Antiquité, Moyen-Âge, réemploi, architecture, décor architectural, rives de l'Ouvèze, «CathédraleNord", cathédrale Notre-Dame-de-Nazareth.

\begin{abstract}
The city of Vaison-la-Romaine offers a study both rich and diversified when talking about the re-employment in architecture. The city is located on the right bank of a river called Ouvèze, following the Roman conquest, which ended in the first half of the first century BC. It was abandoned in the Middle-Ages by the population who migrated to the left of the river bank where a medieval village developed. In modern times, the city once again established itself on the right bank, where the Roman city had been founded, and settled there definitively. The various constructions which succeeded during the different periods on each side of the Ouvèze, have preserved re-employed elements which constitute the precious witnesses of this practice. The three case studies which are talked about are differentiated by their nature and chronology but they attest to the use of the same technique for their implementation. This phenomenon of re-employment invites us to question its process and its reasons as well as the re-employed elements, their new destination and their function, but also their origin.
\end{abstract}

Vaison-la-Romaine, Antiquity, Middle-Ages, re-employment, architecture, architectural ornamentation, river banks of Ouvèze, "Cathédrale-Nord», cathedral Notre-Dame-de-Nazareth.

La ville de Vaison, dont le nom provient du latin Vasio Vocontiorum, soit Vaison des Voconces, se situe au sud-ouest de son territoire. Au ${ }^{\text {er }}$ siècle avant notre ère, elle se développe aux abords de l'Ouvèze. Ce cours d'eau, non navigable et endigué en raison de fortes crues, ne constitue pas une voie de communication fluviale, mais il a joué un rôle majeur dans le développement de l'agglomération. Les premières occupations se concentrent sur la rive gauche puis, à la suite de la conquête romaine qui prit fin durant la première moitié du $\mathrm{I}^{\mathrm{er}}$ siècle avant J.-C., la ville est implantée sur la rive droite. Elle s'y développe considérablement jusqu'au $\mathrm{XII}^{\mathrm{e}}$ siècle avant que la population n'abandonne les lieux pour investir la rive gauche et bâtir le bourg médiéval sur la colline dite du château. La rive droite, qui était fortement urbanisée, est alors complètement désertée et exploitée en terres agricoles jusqu'au XVII ${ }^{\mathrm{e}}$ siècle où une nouvelle migration de la population regagnant celle-ci conduit à l'installation et au développement de la ville moderne ${ }^{1}$. 
L'intérêt pour la ville antique de Vaison et ses vestiges se développa considérablement au cours de cette même période puisque la construction des habitations modernes conduisit à exhumer des vestiges antiques et à attirer l'attention sur ce passé romain. Cependant, ce n'est qu'au début du $\mathrm{XX}^{\mathrm{e}}$ siècle, lors de l'arrivée à Vaison du chanoine J. Sautel (1880-1955), que la compréhension et la valorisation des vestiges prirent véritablement leur importance. Personnalité majeure de la recherche vaisonnaise, il mit au jour pendant toute la première moitié du $\mathrm{XX}^{\mathrm{e}}$ siècle la quasi-totalité des vestiges actuellement visibles et documenta ceux qui sont aujourd'hui perdus ou détruits. Il laissa de nombreux écrits, des plans et des photographies, et surtout des inventaires qui contiennent diverses mentions de blocs architecturaux erratiques ou réemployés ${ }^{2}$, ce qui les rend particulièrement précieux pour cette étude qui s'inscrit dans une réflexion plus générale dans le cadre de recherches doctorales sur l'architecture monumentale et son décor à Vaison-la-Romaine du ${ }^{\text {er }}$ siècle avant J.-C. au III ${ }^{\mathrm{e}}$ siècle après J.- $\mathrm{C}^{3}$.

Le phénomène du réemploi architectural se définit globalement par la mise en ouvre dans un édifice d'éléments provenant d'une construction antérieure. Ces éléments se différencient par leur nature puisqu'il peut s'agir de composantes présentant des formes géométriques simples (de pierres, de briques, de bois, etc.), de revêtements tels que les enduits peints et les placages de marbre, ou bien de pièces ornées ayant pris place, par exemple, dans des ordres. Les raisons du réemploi varient également (économique, esthétique, symbolique) tout comme la destination (construction privée ou publique) ainsi que l'usage nouvellement accordé. L'élément architectural peut être laissé en l'état ou transformé (cassures, retailles, etc.) et il peut recevoir une fonction identique

2. J. Sautel, Vaison dans l'Antiquité (vol. I: histoire de la cité, des origines jusqu'aux invasions Barbares; vol. II: catalogue des objets romains trouvés à Vaison et dans son territoire; vol. III: recueil documentaire illustré), Avignon, 1926.

J. Sautel, Vaison dans l'Antiquité (vol. I: histoire et description de la cité, travaux et recherches de 1927 à 1940; vol. II: catalogue des objets romains à Vaison et dans son territoire; vol. III : recueil documentaire illustré), Avignon, 1941, Lyon, 1942.

3. Recherches entreprises en 2015 dans le cadre d'un doctorat en "Langues, histoire et civilisations des mondes anciens" à l'Université Lumière Lyon 2 sous la direction de J.-Ch. Moretti (CNRS - IRAA). ou distincte de celle qu'il avait dans son premier usage. Ainsi, est-il possible de distinguer le recyclage, le réemploi et la réutilisation. Le premier concerne des blocs architecturaux considérés comme des déchets issus d'une construction vétuste, ayant perdu son utilité ou bien détruite (incendie, séisme, etc.): ils peuvent subir une transformation ou conserver leur configuration originelle. Leur nouvel usage diffère de leur fonction initiale et aucune valeur spécifique ne leur est accordée. Le réemploi se distingue du recyclage pour deux raisons. La première est le choix des matériaux. La récupération peut très bien être effectuée sur des édifices en mauvais état, détruits ou effondrés, mais également sur des monuments encore en élévation impliquant alors une sélection du matériau à réemployer et donc une logique de démantèlement. Le matériau une fois récupéré peut ou non subir une modification, mais sa nouvelle fonction se différencie de la première. Il se distingue du simple recyclage pour une seconde raison: un intérêt est porté au bloc pour sa forme et ses dimensions qui conviennent parfaitement à son nouvel usage, voire pour sa valeur symbolique. Enfin, la réutilisation reprend les mêmes principes que le réemploi et s'en distingue par une mise en ouvre de l'élément architectural identique à ce qu'elle était initialement, avec la même valeur structurelle et ornementale à laquelle peut s'ajouter une valeur symbolique.

Trois cas de réemplois architecturaux ont été identifiés à Vaison, dans la digue de l'Ouvèze, sur le site dit "Cathédrale-Nord" et à la cathédrale Notre-Dame-de-Nazareth, dont l'examen a été récemment entrepris. Leur étude conduit à s'interroger sur la pratique du réemploi et tout ce qu'elle implique et sur la destination première des blocs réemployés.

L'ENDIGUEMENT DE L'OUVÈZE, UN EXEMPLE DE RÉEMPLOI PRÉCOCE

Les premiers travaux sur la rive gauche de l'Ouvèze sont réalisés au cours de l'été 1946 par le chanoine J. Sautel, en aval du pont romain, à la suite d'une importante crue de la rivière (fig. 1 et 2). Des vestiges d'un aménagement d'époque romaine apparaissent sur une centaine de mètres où sont visibles "une série de dalles, appuyées sur des pilotis en bois de chêne et des fragments de 


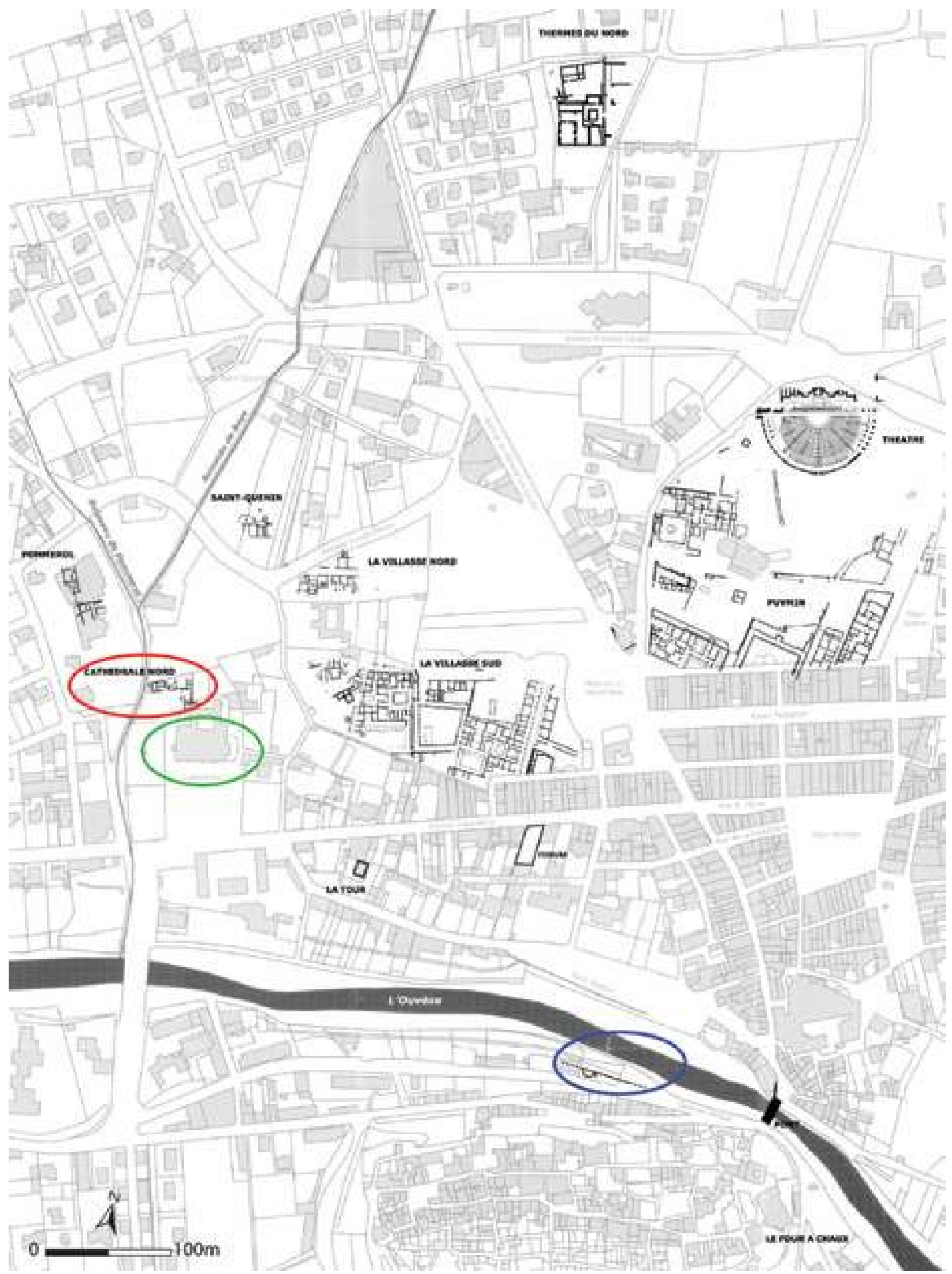

Fig. 1 - Plan de Vaison-la-Romaine avec, en noir, les vestiges antiques connus. En bleu, localisation du mur-digue de l'Ouvèze; en rouge, localisation du site dit «Cathédrale-Nord »; en vert, localisation de la cathédrale Notre-Dame-de-Nazareth (fond de plan - Service du Patrimoine de Vaison-la-Romaine). 


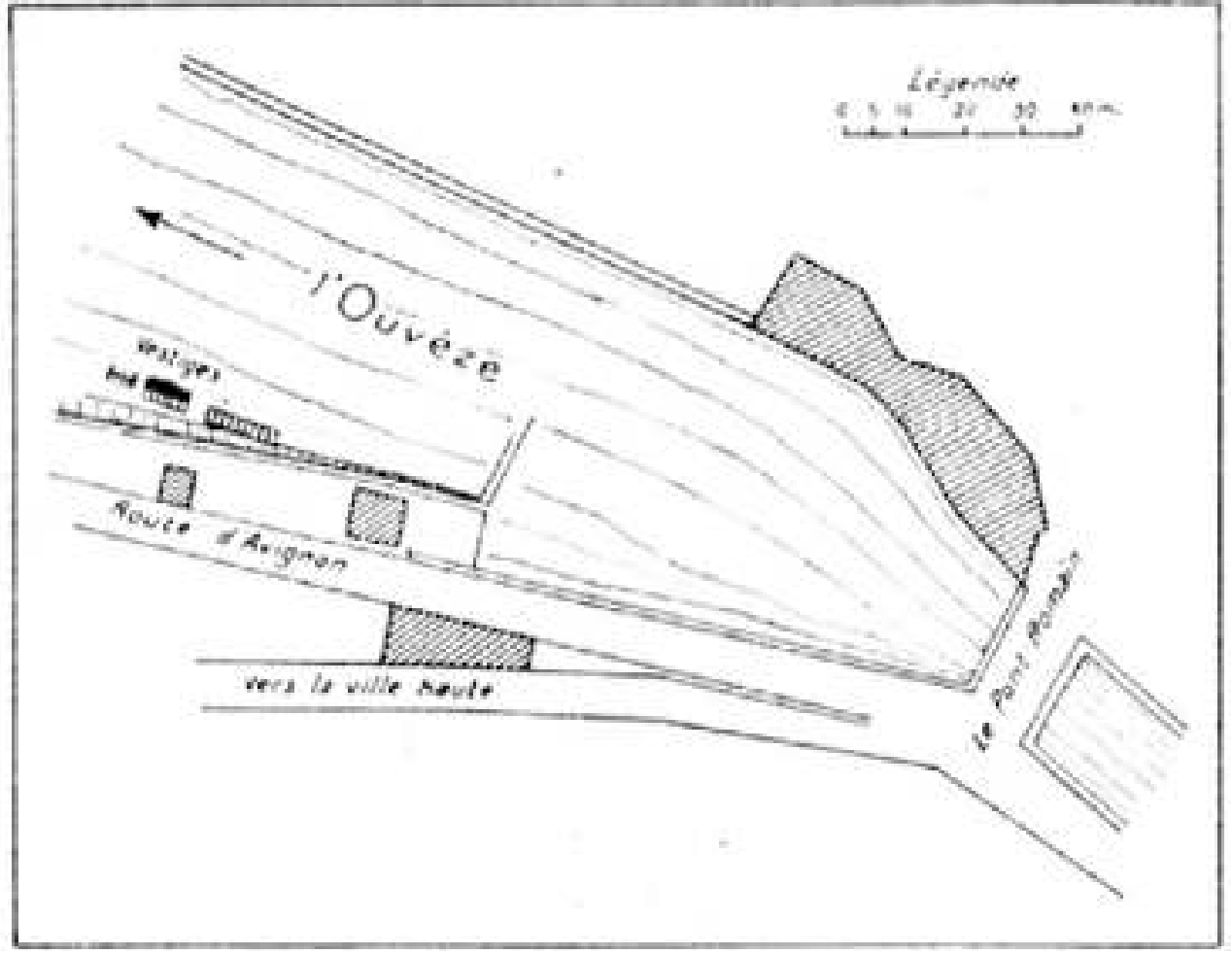

Fig. 2 - Emplacement des vestiges des fondations sur pilotis de la rive gauche de l’Ouvèze. Vue depuis le sud (plan éch. 1/2000e) (fonds J. Sautel, Service du Patrimoine de Vaison-la-Romaine).

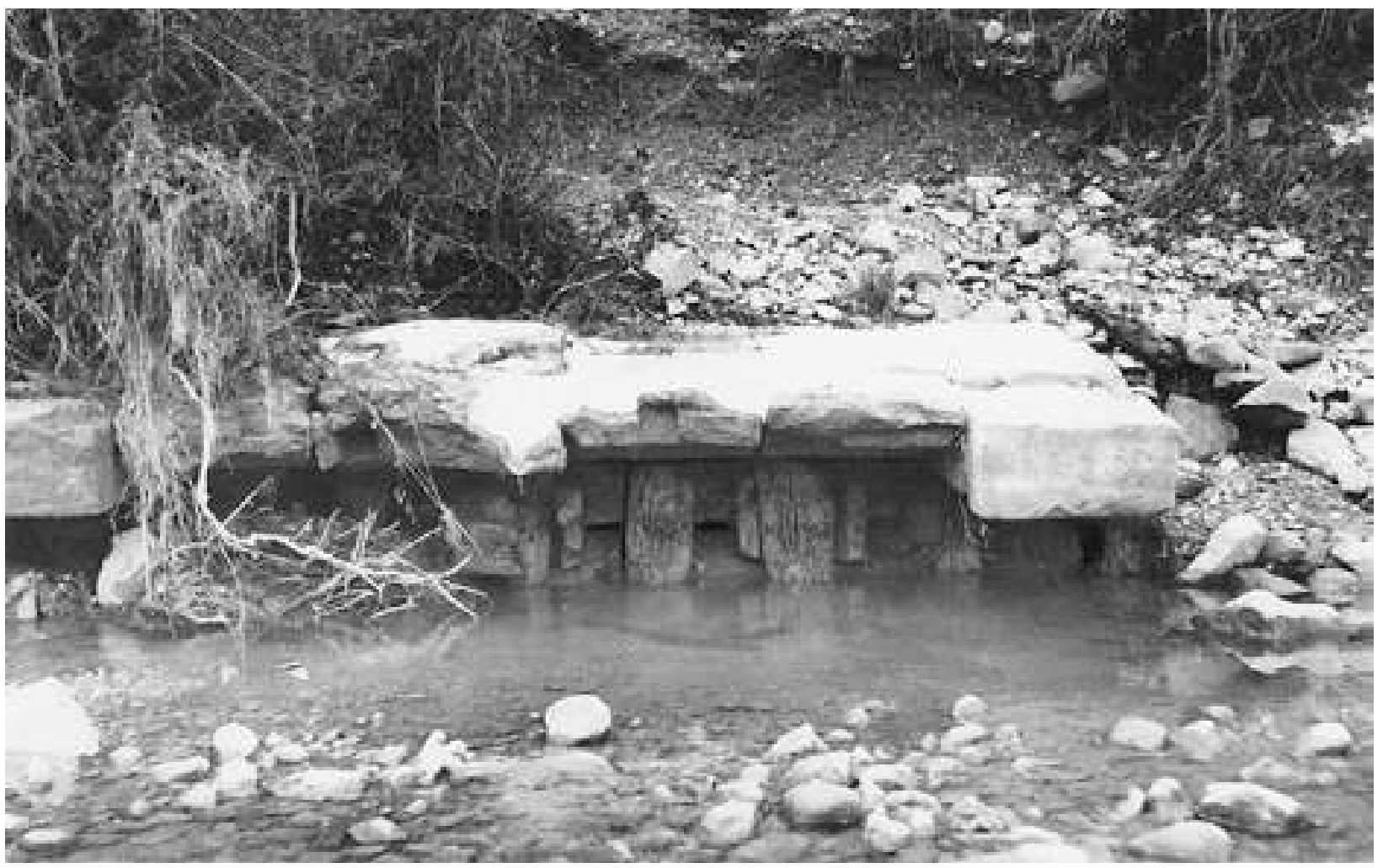

Fig. 3 - Vestiges des fondations sur pilotis de la rive gauche de l'Ouvèze en 1947 (fonds J. Sautel, Service du Patrimoine de Vaison-laRomaine). 
constructions diverses, plus ou moins bouleversées par des glissements de terrain $»^{4}$. Partiellement mis à nus, en particulier grâce à la sécheresse de l'été 1947, ces vestiges font l'objet d'une fouille entreprise par J. Sautel qui reconnaît un dispositif assurant les fondations d'une construction (fig. 3). Il émet l'hypothèse qu'il s'agit d'un mur de soutènement pour une route, des docks ou des entrepôts, ou encore d'un quai pour l'abordage des bateaux notamment ceux des Utricularii de Vaison mentionnés dans une inscription (CIL, XII, $\left.\mathrm{n}^{\circ} 1387\right)^{5}$.

Ce n'est que près d'une cinquantaine d'années plus tard qu'une nouvelle étude est entreprise par l'architecte et archéologue J.-M. Mignon ${ }^{6}$ après la crue du 22 septembre 1992 qui fait réapparaître les vestiges signalés par J. Sautel ensevelis sous les berges ${ }^{7}$. L'année suivante, en septembre 1993, un sondage est réalisé afin d'identifier le dispositif antique et d'effectuer une série de prélèvements sur les pieux pour une analyse dendrochronologique. L'ensemble des observations permit de reconnaître la nature de la construction qui constitue un mur d'endiguement et de fixer une datation pour l'abattage des pieux en chêne à la fin du $\mathrm{I}^{\mathrm{er}}$ siècle de notre ère, et plus précisément en l'année 82 . En 1996, une fouille de sauvetage est entreprise avant la destruction partielle des vestiges engendrée par le tracé d'une nouvelle digue. Le dégagement permet la mise au jour de ce mur-digue sur une longueur de près de $80 \mathrm{~m}$ et qui devait s'étendre à l'origine sur plusieurs centaines de mètres, probablement sur toute la portion urbaine de l'Ouvèze ${ }^{8}$. Seule une partie de la fondation du dispositif observée par J. Sautel a été retrouvée en place. L'élévation de l'extrémité occidentale de la section du mur-digue a été repérée dans le lit de la rivière, basculée dans ce dernier à la suite d'une crue plus ancienne (fig. 4). L'élévation a

4. Sautel 1948, p. 221 ; Mignon 1994, p. 231.

5. Sautel 1926, II, p. 85-86; Sautel 1948, p. 222; Goudineau - de Kisch 1991, p. 131 ; Provost - Meffre 2003, p. 97: "] utric(ulariorum) ci[vitatis (?)». Bien que cette inscription mentionne la présence d'une corporation d'utriculaires à Vaison, l'hypothèse que l'Ouvèze constituait un cours d'eau navigable a été abandonnée depuis les travaux de J.-M. Mignon.

6. Architecte et archéologue au Service d'Archéologie du Département de Vaucluse.

7. Mignon 1994, p. 231.

8. Mignon 1997, p. 168.

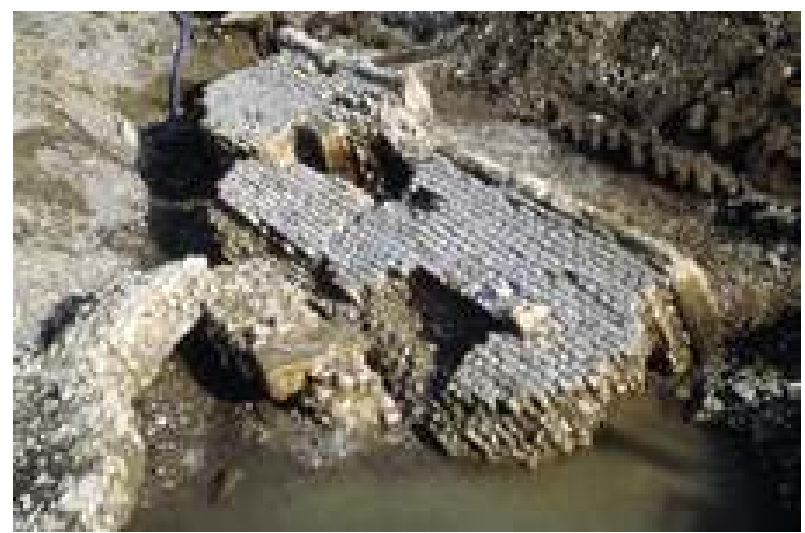

Fig. 4 - Vestiges du mur-digue de la rive gauche de l'Ouvèze dégagés durant les fouilles de 1996 (J.-M. Mignon, Service d’Archéologie du Département de Vaucluse).

une largeur d'environ 2,20 m avec un parement externe en grand appareil du côté de la rivière et un parement interne en petit appareil de moellons. Un intérêt particulier de ce mur-digue réside dans son blocage dans lequel ont été retrouvés des blocs d'architecture fragmentaires réemployés.

L'inventaire récent de ces fragments architecturaux a permis d'en dénombrer trente. Parmi ceux-ci ont été recensés un fragment de base de colonne, un fragment de colonne lisse, des fragments de fûts de colonne cannelés et de pilastres ou de piliers cannelés, un caulicole de chapiteau corinthien, un fragment d'architrave à deux ou trois fasces aux moulurations ornées, trois fragments de corniches ornées dont deux proviennent d'un même élément, quatre fragments de panneaux d'armes dont deux proviennent d'un même élément et d'autres blocs architecturaux ornés dont l'emplacement d'origine n'a pas été déterminé. La nature du réemploi dans le blocage de la maçonnerie semble ici évidente avec le recyclage de ces éléments comme déchets dans leur état ou transformés, consistant en une simple récupération et une utilisation sommaire de ces derniers. La provenance de ces éléments est plus difficile à préciser. La plupart des fragments constituent des blocs d'architecture ornés appartenant à des composantes architecturales bien définies. Sont-ils issus du même édifice ou de plusieurs? Étaient-ils encore en place dans leur architecture primitive et ont-ils été démantelés pour être réemployés? Le ou les édifices auxquels ils appartenaient étaient-ils encore en usage, déjà partiellement dégradés, détruits ou laissés à l'abandon? 


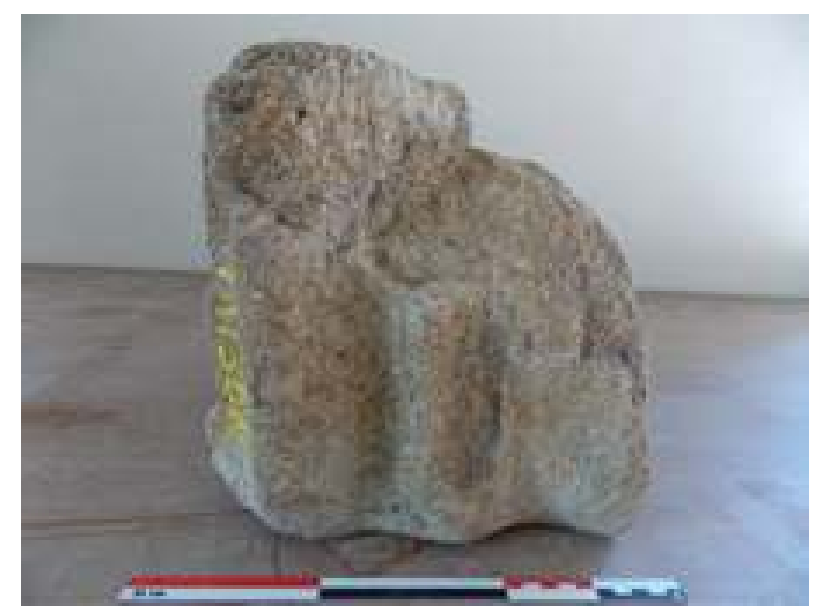

Fig. 5 - Fragment d'un fût cannelé de colonne d'une hauteur complète de $26,3 \mathrm{~cm}$ ( $\mathrm{n}^{\circ}$ inv. : VAI.RIV.96.25) (C. Lefebvre).

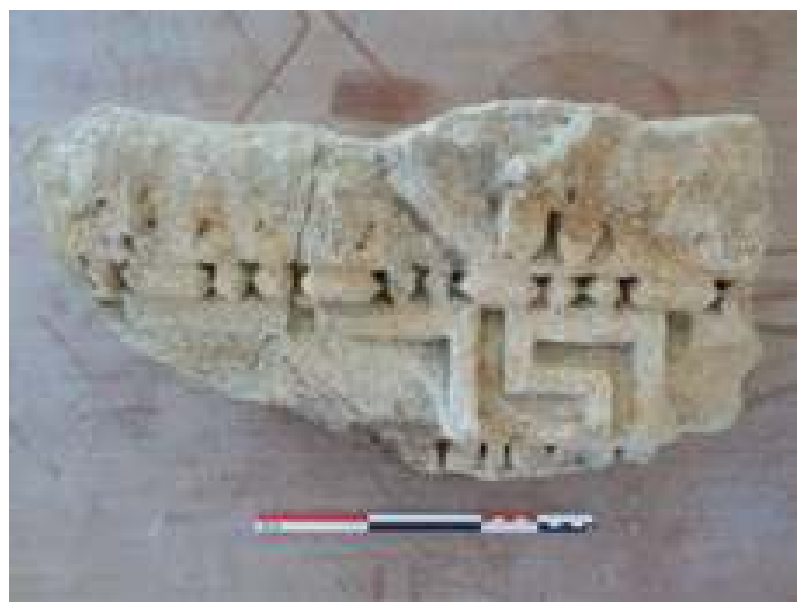

Fig. 6 - Deux fragments jointifs d'un bloc de corniche ornée à larmier ( $\mathrm{n}^{\circ}$ inv. : VAI.RIV.96.24) (C. Lefebvre).

Un des apports considérables de la fouille de 1996 est la datation livrée par l'analyse dendrochronologique des pieux en bois de la fondation du mur-digue. L'abattage du bois évalué autour de 82 impose ainsi un terminus post quem pour l'érection du mur-digue et, bien que moins précisément, un terminus ante quem pour les fragments réemployés impliquant qu'ils appartenaient à un ou plusieurs édifices déjà existants avant cette date. L'analyse typologique permet non pas d'apporter une solution mais seulement quelques précisions. Parmi les divers éléments cannelés, l'analyse a permis de distinguer deux ensembles architecturaux: cinq fragments constituent des assises de pilastres ou de piliers cannelés et quatre autres appartiennent à des fûts de colonnes cannelés dont un constitue un tambour avec une hauteur complète de $26,3 \mathrm{~cm}$ (fig.
5). Les faibles dimensions de ce tambour laissent penser qu'il provient d'un édifice non monumental. Un dernier élément, le plus orné de tous, nécessite une certaine attention. Il s'agit d'un bloc de corniche ornée à larmier formé par deux fragments jointifs (fig. 6). Il comporte plusieurs moulurations animées chacune de différents motifs ornementaux: en partie inférieure sur le talon prennent place des rais-de-cour en ciseau; au-dessus le front du larmier est orné de méandres "en gamma 1 " ${ }^{9}$ à gauche; le quart-de-rond qui le surmonte comporte un rang de perles et de pirouettes; un second talon droit comporte à nouveau des rais-de-cœur dégradés mais probablement en ciseau. Trois motifs se distinguent mais ils ne fournissent pas d'indices chronologiques révélateurs. Le rai-de-cœur en ciseau comme décor du talon est habituel, ce qui est notamment le cas à Vaison sur les talons de couronnement de nombreux éléments d'architraves et de corniches. Ce motif était en usage à Rome dès l'époque augustéenne mais semble surtout s'être diffusé à la fin de la période julio-claudienne et à la période flavienne, ce qui se retrouve également en Narbonnaise ${ }^{10}$. Le méandre "en gamma 1 " apparaît ici, pour la première fois, sur un bloc du corpus vaisonnais. Comme ornement de front de larmier, ce motif semble attesté tout au long du $\mathrm{I}^{\text {er }}$ siècle aussi bien en Italie qu'en Narbonnaise ${ }^{11}$. Ces motifs n'apportent pas véritablement de données inédites sur la nature du ou des édifices desquels ils proviennent. Ils suggèrent tout de même une datation au $\mathrm{I}^{\mathrm{er}}$ siècle de notre ère sans plus de précision au vu du peu de motifs décoratifs.

Lors de la découverte des fragments, J.-M. Mignon avait suggéré de les restituer à un édifice funéraire de type mausolée d'époque augustéenne ou flavienne en raison du terminus ante quem de 82 mais également grâce à la présence d'une quantité importante de céramiques romaines datées du $\mathrm{I}^{\mathrm{er}}$ siècle de notre ère ${ }^{12}$. L'hypothèse est recevable au vu du format des fragments réemployés et également du motif des panneaux d'armes ornés de boucliers qui est souvent attesté dans l'architecture funéraire (fig. 7).

9. Ginouvès - Martin 1985, p. 181 et pl. 52.2.

10. Fellague 2007, p. 668; à la période augustéenne se rencontre plus souvent le rai-de-cœur en étrier (Tardy 1994, p. 117, note 113).

11. Fellague 2007, p. 683-684.

12. Mignon 1997, p. 169. 


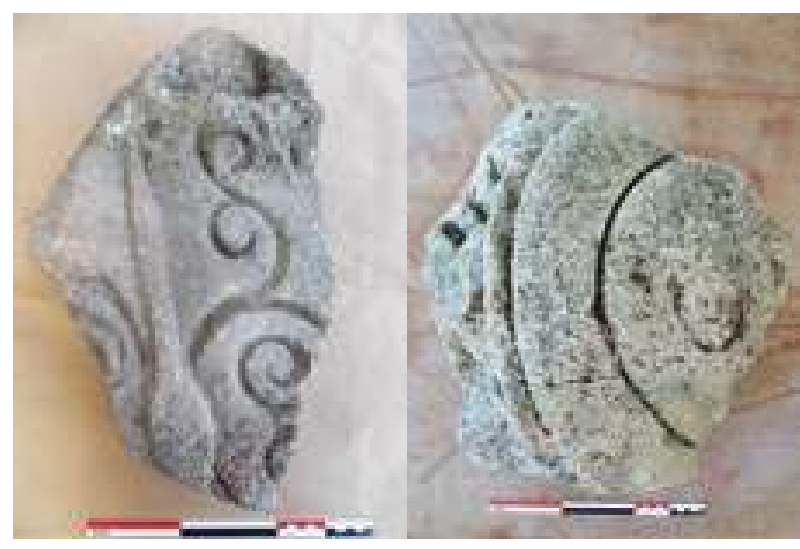

Fig. 7a-b - Deux fragments de panneaux d'armes ornés de boucliers ( $\mathrm{n}^{\circ}$ inv. : VAI.RIV.96.11; VAI.RIV.96.16) (C. Lefebvre).

DES RÉEMPLOIS DANS L'ANTIQUITÉ TARDIVE? LE SITE DIT «CATHÉDRALE-NORD »

Situé au nord de la cathédrale Notre-Damede-Nazareth et de son cloître (fig. 1), ce site a fait l'objet d'importantes fouilles de 1972 à 1981. En vue de l'aménagement d'un parking, des fouilles furent engagées sous la direction de B. Liou ${ }^{13}$ qui les conduisit jusqu'en 1978. Elles furent reprises l'année suivant par A. Kauffmann ${ }^{14}$ qui les poursuivit jusqu'en 1980. Y. Roumégoux et Fr. BartaudGalliou terminèrent les dégagements en 1981. Le site fut ensuite délaissé en l'état jusqu'en 1993 où il fut enfin remblayé sous l'initiative du S.R.A. P.A.C.A ${ }^{15}$.

Au nord-ouest du site, des sondages réalisés en 1972 et 1973 avaient permis la reconnaissance de murs datés entre le $\mathrm{IV}^{\mathrm{e}}$ et le $\mathrm{VI}^{\mathrm{e}}$ siècle (fig. 8). $\mathrm{Au}$ sein de ces constructions, des éléments architecturaux réemployés avaient pu être observés. La fouille de cette partie du site entre 1978 et 1980 révéla la présence d'un grand nombre de réemplois dans les blocages de fondations ou de murs, et parfois laissés visibles dans les parements de ces derniers. Certains d'entre eux sont toujours sur le

13. En collaboration avec E. Deniaux, Y. de Kisch, P. Thollard, J.-M. Gassend et A. Barbet.

14. Je profite de cette occasion pour adresser mes sincères remerciements à Monsieur A. Kauffmann qui m'a permis de consulter l'ensemble de ses archives comprenant notamment les fiches d'inventaire et les photographies des blocs réemployés, témoignages plus que précieux pour mener à bien cette nouvelle étude.

15. Provost - Meffre 2003, p. 134.

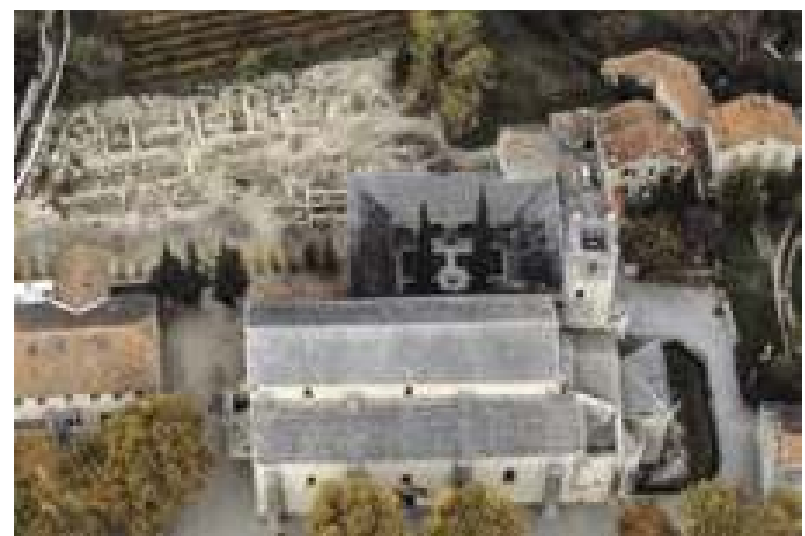

Fig. 8 - Vue aérienne du site dit "Cathédrale-Nord». Vue depuis le sud (D. Carru, Service d'Archéologie du Département de Vaucluse).

site, aujourd'hui enseveli, mais d'autres ont été prélevés lors du démontage des structures et ont été déposés au dépôt archéologique de la Villasse à Vaison-la-Romaine.

Parmi les éléments laissés en place apparaissent des blocs de piliers à pilastres engagés tous deux cannelés, ainsi que de grands blocs quadrangulaires lisses simplement accolés les uns aux autres sans liant particulier (fig. 9). Les premiers avaient été réemployés sur deux rangées d'assises pour constituer les soubassements d'un espace nommé de fait "la salle au gros soubassements ${ }^{16}$. Les photographies d'époque attestent le bon état de conservation de ces imposants éléments architecturaux. Les cannelures des faces de parement sont nettement lisibles et les lits de pose et d'attente parfaitement dressés, ces derniers disposant en leur centre de trous de louve témoignant de leur mise en ouvre dans leur architecture initiale. Le réemploi semble donc ici répondre à des besoins d'ordre pratique avec l'usage d'éléments de grandes dimensions et de forme rectangulaire permettant la construction de fondations dont la largeur est définie par celles des blocs réemployés. Ces derniers ne présentent pas de traces de retailles ou d'importantes cassures, le format des blocs correspondant parfaitement à leur nouvelle destination. La non nécessité de les retravailler ajoute à la notion pratique un intérêt bien évidemment économique puisque le matériau est disponible et prêt à l'emploi. L'état des éléments, leurs dimensions conservées ainsi que

16. Kauffmann 1979, p. 8-9; Provost-Meffre 2003, p. 142. 
leur réemploi en un unique endroit du site et au sein d'une même structure invitent à s'intéresser à leur récupération suggérant l'idée d'un démantèlement dans le but d'une réutilisation immédiate. Malheureusement, la nature de l'édifice dont l'élévation aurait été spoliée est inconnue. En 1980, Y. Roumégoux et M.-Fr. Ribaud avaient émis l'hypothèse que les assises de piliers à pilastres engagés constituaient initialement «les pierres d'un arc monumental antique ${ }^{17}$, les pilastres correspondant aux piédroits d'un arc surmontés d'impostes. Cette hypothèse ne renseigne pas sur l'identification de l'édifice dans sa globalité, mais seulement sur une partie de son architecture. Elle paraît, à nos yeux, probable et se trouve étayée par l'étude des fragments prélevés que nous avons récemment réalisée.

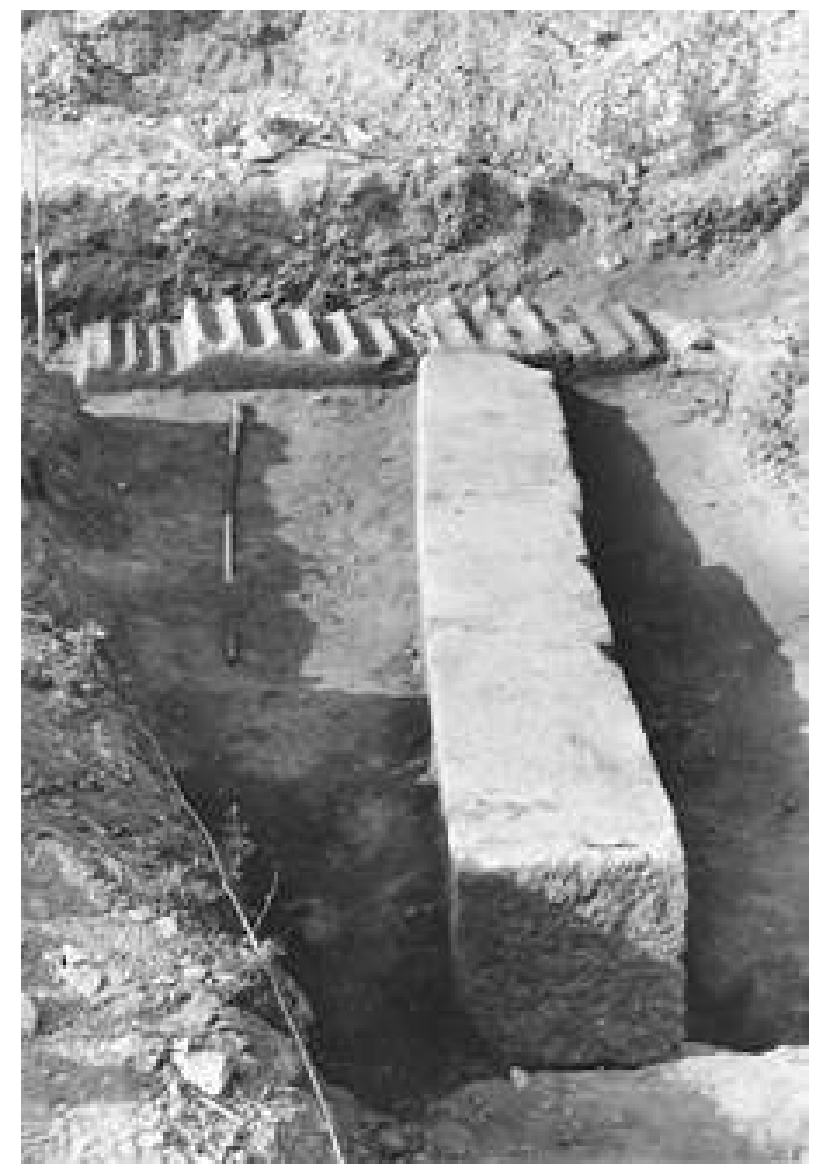

Fig. 9 - Blocs de piliers à pilastres engagés tous deux cannelés et blocs quadrangulaires lisses découverts lors des sondages de 1973 sur le site dit "Cathédrale-Nord » (B. Liou, Service Régional de l'Archéologie DRAC-PACA).

17. Provost - Meffre 2003, p. 142-143.
Il s'agit de quarante-et-un fragments qui ont été extraits non pas de "la salle aux gros soubassements", mais de plusieurs murs de même datation et situés à quelques mètres au nord de celle-ci. D'état fragmentaire et de dimensions très réduites pour certains, ils prenaient place dans le blocage des fondations ou des élévations, ou parfois disposés dans les parements. Ils constituent assurément ici des réemplois en tant que déchets comme cela a déjà pu être constaté précédemment avec l'étude du mur-digue de l'Ouvèze. Contrairement aux blocs de piliers utilisés en soubassement, le format initial des éléments n'a pas été pris en compte dans la pratique du réemploi. Les faces de parement ornées et très bien conservées pour nombre d'entre eux n'ont pas été retenues pour leur valeur ornementale. Seule une minorité apparaît dans les parements des murs. Les traces de mortier visibles sur celles-ci et les faces cassées témoignent d'un inintérêt pour leurs motifs ornementaux. Mis à part trois fragments d'acrotère, trois fragments de panneaux d'armes, un fragment de sculpture tous en calcaire coquiller de Beaumont-du-Ventoux, ainsi que quelques éléments d'applique en marbre blanc à grain fin dont un chapiteau corinthien, deux fragments d'une même architrave à deux ou trois fasces ainsi qu'un couronnement, l'ensemble des éléments fragmentaires provient de composantes architecturales qui constituaient l'élévation d'un ou plusieurs édifices. Parmi eux, peuvent être rapidement cités des fûts de colonnes lisses ou cannelés, des chapiteaux d'ordre ionicisant et corinthien et de nombreux claveaux d'arcs aux archivoltes ornées. La majorité sont en calcaire coquiller de Beaumont-du-Ventoux, pierre fréquemment employée dans la construction monumentale romaine à Vaison et certains en calcaire beige à grain fin dont la provenance est encore inconnue. Treize de ces éléments constituent des claveaux fragmentaires appartenant à deux arcs distincts aux archivoltes ornées, quatre pour le premier et sept pour le second. C'est notamment la présence de ces deux arcs incomplets qui permet d'appuyer, à notre sens, l'hypothèse émise en 1980 concernant les assises de piliers à pilastres engagés composant les piédroits d'un arc. L'étude typologique des treize fragments assure l'existence de deux arcs sans que l'on puisse savoir à quel type d'édifice ils appartenaient.

Le premier arc (fig. 10) présente une archivolte ornée dans sa partie inférieure d'une alternance de 
motifs végétaux assez mal conservés. Au-dessus, se développent de bas en haut une file de perles ornant un astragale, une torsade à deux brins avec oeillets sans bouton ornant un second astragale et enfin le couronnement de l'archivolte qui présente de nouveaux motifs végétalisés de type rinceaux. L'astragale de perles se retrouve traditionnellement dans le vocabulaire décoratif gallo-romain employé à partir de l'époque flavienne et au II ${ }^{\mathrm{e}}$ siècle, notamment dans la seconde moitié, mais certains exemples sont déjà connus dès l'époque augustéenne ${ }^{18}$. Les tresses à deux brins qui ornent un astragale et présentent un profil convexe avec un œeillet laissé vide entre chaque entrelacement (sans bouton), constituent une variante largement diffusée au I ${ }^{\text {er }}$ siècle en Narbonnaise et particulièrement à l'époque julio-claudienne ${ }^{19}$. Bien que cette variante de tresse semble attestée au ${ }^{\text {er }}$ siècle, il serait imprudent de dater ces quatre fragments à partir de ce seul motif.

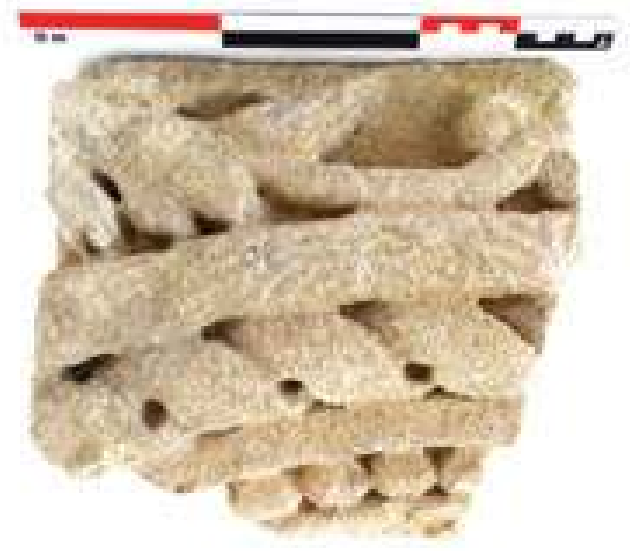

Fig. 10 - Un des quatre fragments de claveaux qui composent le premier arc orné découvert sur le site dit "Cathédrale-Nord 》 ( ${ }^{\circ}$ inv. : VAI.CATH.NORD 79.14) (C. Lefebvre).

18. Tardy 1994, p. 131; Fellague 2007, p. 674.

19. Cette variante est «au I ${ }^{\text {er }}$ siècle, de loin la plus fréquente en Narbonnaise, tout comme en Aquitaine où, particulièrement à l'époque julio-claudienne, elle est employée tant au couronnement d'abaque des chapiteaux corinthiens et composites, qu'en décor d'astragale, notamment en association avec les motifs de rais-de-cœur en ciseau et les godrons " (Tardy 2005, p. 115).

Tardy 1994, p. 136: "Ainsi, le type A [tresse à deux brins sans oeillet], dans sa version saintaise, s'inscrit dans une série d'époque julio-claudienne, bien représentée sur les monuments de Narbonnaise, où, sur astragale, il constitue un décor de moulure de transition".
Le second arc dont sept fragments ont été reconnus (fig. 11) a des motifs ornementaux bien lisibles, mais souvent de manière ponctuelle. Cela permet toutefois de retrouver la succession des moulures qui ornaient l'archivolte et de distinguer certains décors tels que les files de perles en décor d'astragale, un rang d'oves et fers de lance en décor d'un ovolo et une alternance de motifs végétaux toutefois incomplets (rinceaux, feuilles d'acanthe, palmette flammée ouverte). Pour la chronologie des oves et fers-de-lance, il est communément admis ${ }^{20}$ que le passage de l'époque julio-claudienne à la période flavienne est marqué par un remplacement des fers de lance par des pointes de flèche, changement qui s'opère une nouvelle fois et en sens inverse plus tardivement dans la première moitié du $\mathrm{II}^{\mathrm{e}}$ siècle. Les indices chronologiques livrés par les oves et fers-de-lance coïncident avec ceux des files de perles que l'on observe durant tout le I ${ }^{\text {er }}$ siècle et durant la seconde moitié du $\mathrm{II}^{\mathrm{e}}$ siècle. L'analyse typologique de ces motifs oriente donc vers une fourchette chronologique assez large.

Un tout autre motif ornemental a été observé sur un fragment de chapiteau corinthien de colonne (fig. 12). Ce dernier, dont seule une partie du registre supérieur du calathos est conservée, comporte une hélice dont la tige est totalement recouverte d'une feuille d'acanthe. Cette particularité qui devait également apparaître sur les tiges des volutes est caractéristique du corinthien tardo-augustéen et d'une partie de la période julio-claudienne témoignant $\mathrm{d}^{\prime}$ «une curieuse réminiscence d'une pratique décorative propre à l'époque du second triumvirat $»^{21}$. Elle est toutefois attestée jusqu'à l'époque flavienne en milieu provincial, ce qui étend la datation à tout le $\mathrm{I}^{\mathrm{er}}$ siècle ${ }^{22}$. La datation suggérée par la présence de "Fiederblätter" concorderait avec celles des différents motifs ornant les deux arcs pour le $\mathrm{I}^{\text {er }}$ siècle et notamment la période julio-claudienne. Toutefois, nous ne connaissons pas les provenances de la

20. Tardy 1994, p. 122-123; Fellague 2007, p. 658.

21. Gros 1979, p. 73, note 70; Tardy 2005, p. 60.

En effet, au cours de la dernière décennie avant notre ère, ces "Fiederblätter" avaient été abandonnés sur les chapiteaux italiens et provinciaux et refont leur apparition au changement d'ère sur divers exemplaires d'Italie et des provinces.

22. Tardy 1989, p. 80. 

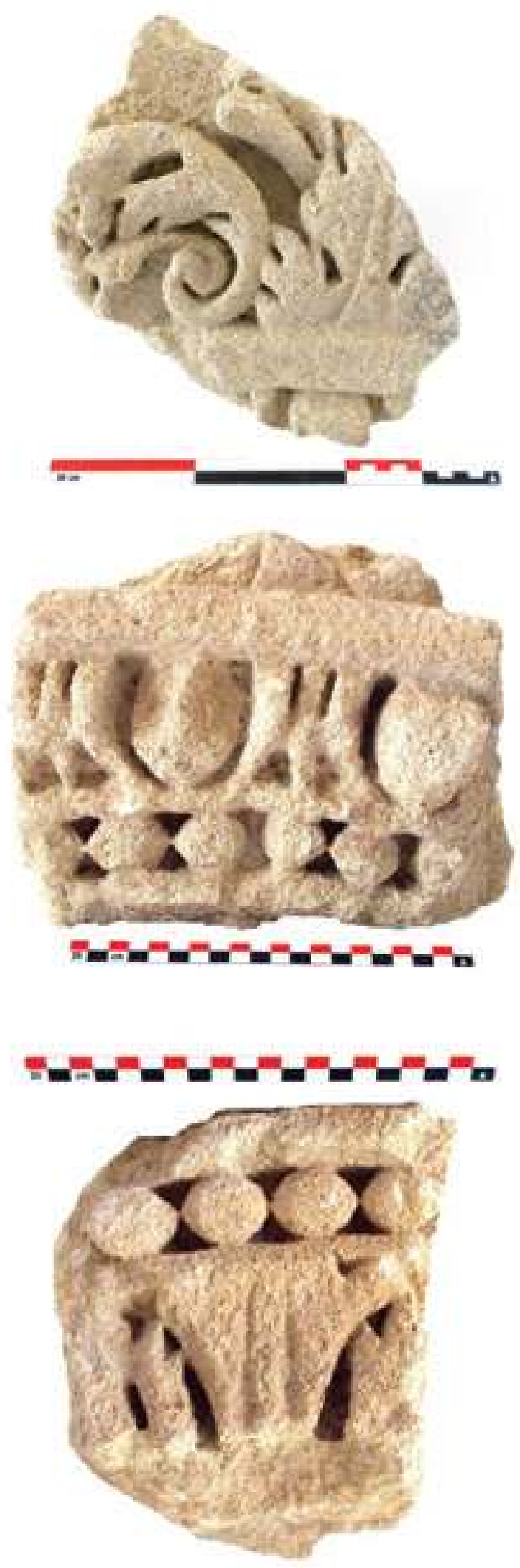

Fig. $11 \mathrm{a}-\mathrm{c}$ - Trois fragments de claveaux constituant le second arc orné découvert sur le site dit "Cathédrale-Nord" ( $n^{\circ}$ inv. : VAl.CATH. NORD 82.5, 82.9 et 79.3) (C. Lefebvre). totalité de ces fragments réemployés qui peuvent ne pas appartenir au même ensemble architectural. En effet, ce type de feuille de recouvrement apparaît également sur un deuxième fragment de chapiteau corinthien (fig. 13) dont les dimensions suggèrent qu'il n'appartenait pas à une construction monumentale. Plusieurs édifices ont donc été spoliés, monumentaux ou plus modestes, et de nature publique ou bien privée (habitat ou funéraire).

\section{LA CATHÉDRALE NOTRE-DAME-DE-NAZARETH, UN CAS D'ÉTUDE PARTICULIER}

La cathédrale Notre-Dame-de-Nazareth de Vaison a été érigée sur la rive droite de l'Ouvèze là où s'était développée la ville romaine (fig. 1). L'étude de cet édifice, entreprise par C. Michel $\mathrm{d}^{\prime}$ Annoville ${ }^{23}$ en $1993^{24}$, a mis en évidence la succession de trois constructions: un premier bâtiment paléochrétien, un second postérieur au $\mathrm{VI}^{\mathrm{e}}$ siècle et un dernier bâti aux $\mathrm{XI}^{\mathrm{e}}$ et $\mathrm{XII}^{\mathrm{e}}$ siècles, l'actuelle cathédrale romane ${ }^{25}$. C'est cette dernière qui entre dans le cadre de notre propos sur le réemploi. Entre 1949 et 1953 le monument religieux fait l'objet de sondages et de fouilles par le chanoine J. Sautel qui dégage rapidement, au niveau du chevet de la cathédrale, les fondations de l'abside centrale encastrée dans un massif quadrangulaire à l'extérieur du monument et celles des absidioles qui l'encadrent au nord et au sud. Cette opération met au jour l'ensemble de la fondation intégralement bâtie à partir blocs architecturaux de monuments romains réemployés ${ }^{26}$ (fig. 14). J. Sautel relève la présence de "trois moitiés de chapiteaux, d'un diamètre complet de $0,70 \mathrm{~m}$ et de $0,90 \mathrm{~m}$ et d'une hauteur moyenne de $0,50 \mathrm{~m}$ » et de «vingttrois tambours de colonnes cannelées de $0,70 \mathrm{~m}$ à $0,90 \mathrm{~m}$ de diamètre et d'une hauteur variant entre $0,60 \mathrm{~m}$ et $0,85 \mathrm{~m} »^{27}$. Il émet l'hypothèse que l'édifice romain en question était «un grand monument du ${ }^{\text {er }}$ siècle, qui devait être un temple et

23. Professeur, Université Paris IV - Sorbonne.

24. C. Michel d'Annoville, La cathédrale Notre-Dame-de-Nazareth, étude des sondages du chanoine Sautel, rapport de sauvetage urgent, S.R.A. P.A.C.A., 1993.

25. Michel d'Annoville 1994, p. 230-231.

26. Sautel 1950a, p. 9; Sautel 1950b, p. 165; Salet 1951, p. 79.

27. Sautel 1950a, p. 9. 


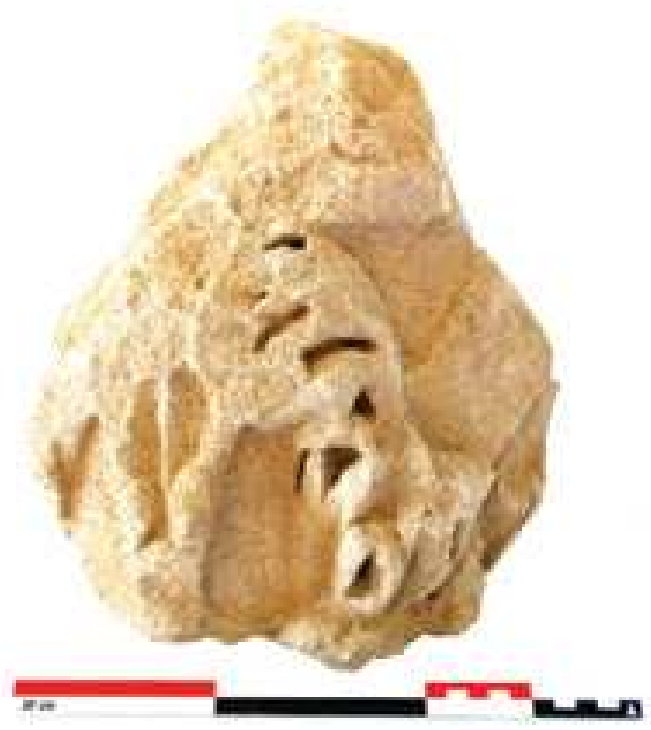

Fig. 12 - Fragment d'un chapiteau corinthien de colonne d'ordre monumental avec "Fiederblätter» ( ${ }^{\circ}$ inv. : VAI.CATH.NORD 80 (1)) (C. Lefebvre).

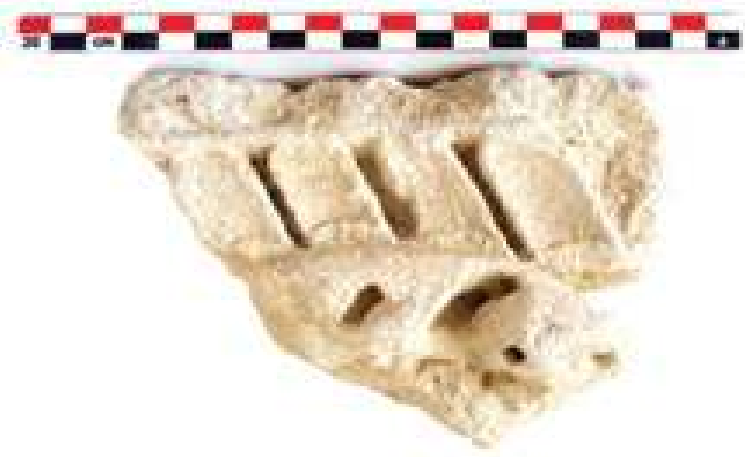

Fig. 13 - Fragment d'un chapiteau corinthien de modestes dimensions avec "Fiederblätter» ( ${ }^{\circ}$ inv. : VAI.CATH.NORD 81.3) (C. Lefebvre).

qui était situé dans le voisinage $»^{28}$. Dernièrement, une mission archéologique nommée "Vaison 3D " menée en mai et juin 2016 pour le relevé 3D de la cathédrale Notre-Dame-de-Nazareth sous la direction de C. Michel d'Annoville, a relancé l'étude du monument et celle des réemplois architecturaux observés par J. Sautel.

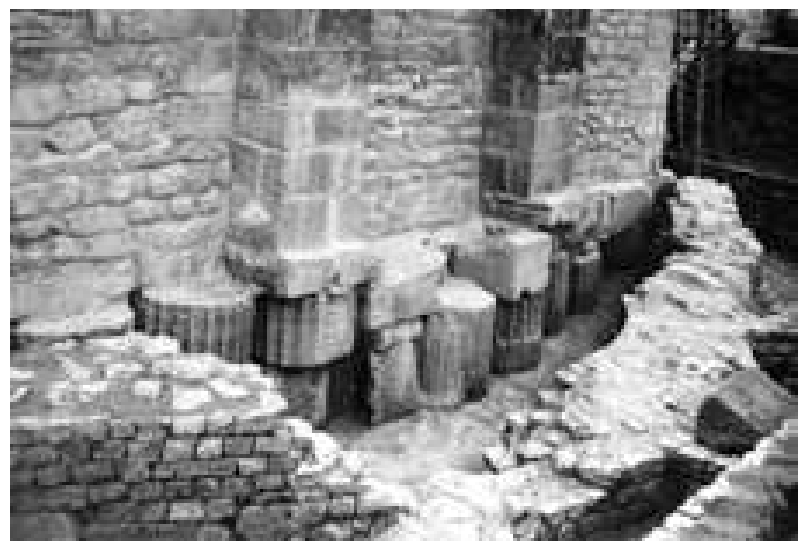

Fig. 14 - La fondation du massif du chevet de la cathédrale NotreDame-de-Nazareth prise à la suite de son dégagement en 1949 par le chanoine J. Sautel (fonds J. Sautel, Service du Patrimoine de Vaison-la-Romaine).

Parmi les éléments remployés dans les fondations prennent place des pierres de taille de grand appareil aux faces taillées et lisses sans ornementation. Vingt-sept blocs d'architecture ont également été identifiés dont vingt-cinq constituent des composantes d'ordre. Deux éléments architecturaux moulurés ont été enregistrés, possible chaperon et linteau de porte, ainsi qu'un fragment de fût de colonne lisse, vingt-et-un tambours de fûts de colonne cannelés dont trois sont cannelés et rudentés, et trois moitiés de chapiteaux corinthiens (fig. 15). L'ensemble des éléments a été taillé dans le calcaire coquiller de Beaumont-duVentoux, principal matériau utilisé dans l'architecture monumentale de la ville dont les carrières se situent à une dizaine de kilomètres de Vaison-laRomaine.

La disposition de ces blocs témoigne d'une certaine organisation lors de la mise en place de la fondation. Durant le dégagement de 1949, J. Sautel avait observé que les blocs étaient "fixés sur des pierres de petit appareil au moyen d'un mortier grossier et peu résistant ${ }^{29}$. Leurs faces externes sont alignées et délimitent le massif central quadrangulaire ainsi que les deux absidioles semi-circulaires nord et sud du chevet. Les blocs reposent en deux assises, parfois trois au niveau des contreforts qui renforcent l'élévation du chevet. En effet, sous ces derniers prennent place des blocs servant de soubassement. Les éléments qui constituent les deux premières assises sont irrégulière- 

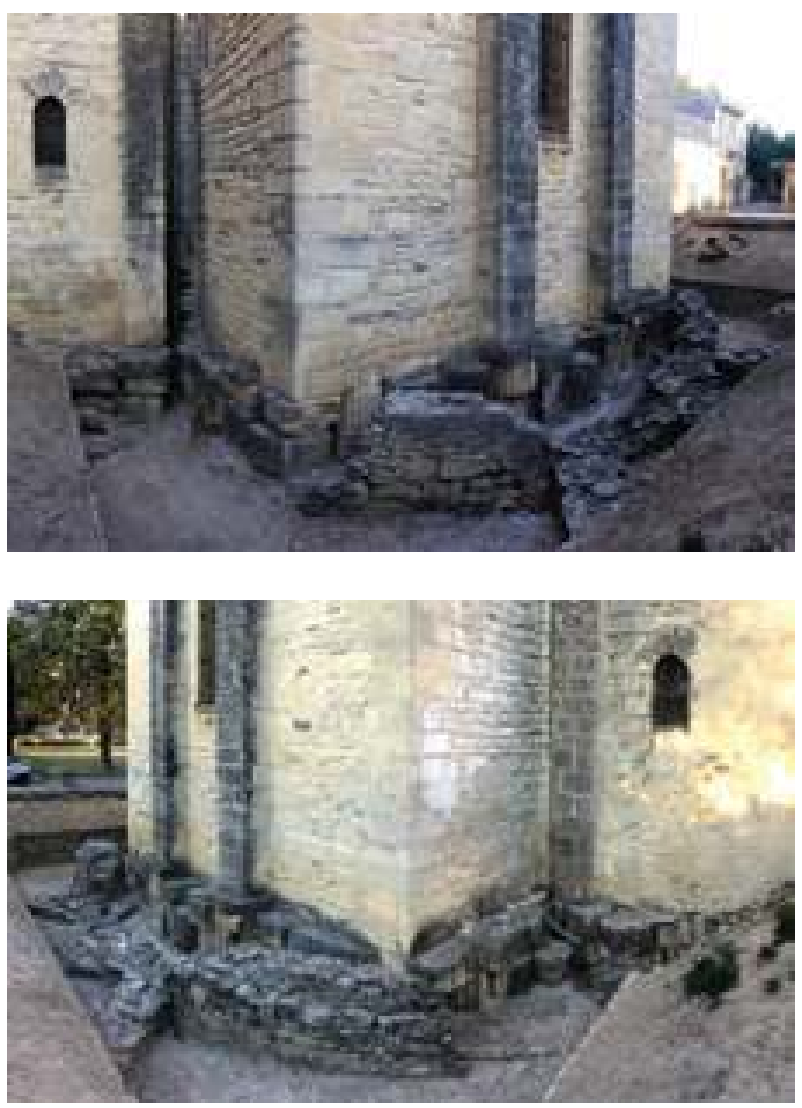

Fig. 15a-b - Les réemplois de blocs architecturaux antiques dans la fondation du chevet de la cathédrale Notre-Dame-de-Nazareth en 2016 (C. Lefebvre).

ment espacés. Cela laisse entrevoir dans les interstices une seconde rangée à deux assises également formées de réemplois disposés sur l'exemple de la première et masquée par celle-ci. La réutilisation d'éléments architecturaux s'étend donc à l'ensemble de la fondation du chevet. Cette donnée avait déjà été affirmée par J. Sautel en 1950. Cette année-là, il entreprend des sondages à l'intérieur de la cathédrale au niveau de l'abside semi-circulaire centrale. Il met au jour, sous le dallage, un niveau plus ancien correspondant à celui des fondations du chevet et constitué de réemplois d'éléments architecturaux romains identiques à ceux qui ont été dégagés à l'extérieur du monument ${ }^{30}$. Ces

30. Sautel 1950-1951, p. 203: "Celles [les fouilles] qui ont été faites en 1950 à l'intérieur de l'abside principale ont confirmé ces conclusions. En effet, après avoir défoncé le dallage récent, les ouvriers ont atteint un niveau plus ancien, qui correspond à celui des fondations extérieures, indiqué par les débris romains en réemploi découverts l'année précédente (...)». éléments témoignent d'une disposition réfléchie pour la mise en place de la fondation. Bien qu'ils n'aient pas été réutilisés en tant que composantes architecturales, ils n'ont pas été considérés comme de simples déchets dans un processus de recyclage. La grande majorité présente un très bon état de conservation avec de rares traces de brisures ou de retailles volontaires, leur forme initiale servant parfaitement à leur nouvel usage. La conservation des blocs dans leur intégralité ainsi que leur nombre invitent à s'interroger sur le processus du réemploi. Il semble possible que les blocs aient été encore en place dans leur édifice d'origine et qu'ils aient été démantelés spécifiquement pour constituer la fondation du chevet. Cette hypothèse soulève d'autres interrogations telles que la sélection des éléments destinés au réemploi et la mise en place d'un chantier de récupération précédant le chantier de construction, ainsi que l'identification du ou des monuments romains spoliés.

La provenance des différents blocs d'architecture inventoriés est pour le moment inconnue. L'étude typologique débutée récemment et toujours en cours ne permet pas d'en savoir plus dans l'état actuel. Elle permet toutefois, par comparaison avec le corpus vaisonnais déjà établi, de savoir que ces tambours de colonnes cannelés sont les exemplaires les plus importants par leurs dimensions connus à Vaison. En outre, ils se répartissent assurément en deux ensembles, l'un avec des fûts de colonnes à 20 cannelures (l. cannelure: 8,5 à 9,5 cm; prof. cannelure: 2,5 à $3 \mathrm{~cm}$ ) d'un diamètre de $70 \mathrm{~cm}$ et l'autre avec 24 cannelures (l. cannelures: 8 à $9 \mathrm{~cm}$; prof.: $4 \mathrm{~cm}$ ) et d'un diamètre de $90 \mathrm{~cm}$ (fig. 16 et 17). La présence de tambours cannelés ou cannelés et rudentés qui constituent les extrémités inférieures et supérieures des fûts permettra probablement, en fonction des hauteurs et des diamètres des tambours, d'évaluer et de restituer la hauteur complète des fûts. Parmi les trois chapiteaux corinthiens de colonne (fig. 18 et 19), l'un constitue le registre inférieur d'un calathos corinthien et les deux autres les registres supérieurs surmontés de l'abaque. L'analyse typologique de chaque motif ornemental n'a pas encore été réalisée mais la facture générale laisse avancer une datation dans le $\mathrm{I}^{\text {er }}$ siècle de notre ère ${ }^{31}$.

31. Celle-ci résulte du découpage asymétrique des feuilles d'acanthe du calathos, des zones d'ombre en forme de 


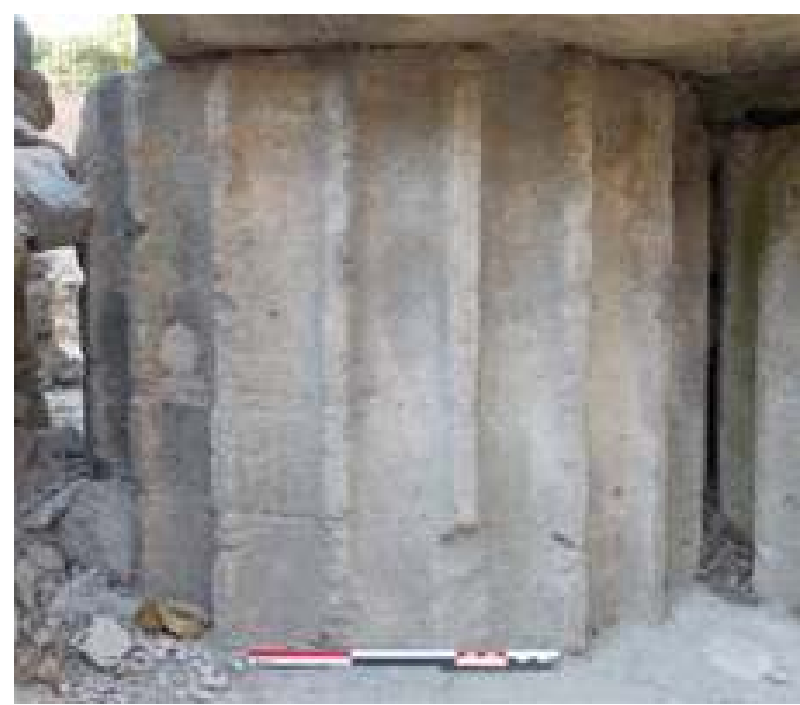

Fig. 16 - Tambour d'un fût cannelé de colonne orné de 20 cannelures et d'un diamètre de $70 \mathrm{~cm}$, réemployé dans la fondation du chevet de la cathédrale Notre-Dame-de-Nazareth ( $n^{\circ}$ inv. : VAl. CATH.19) (C. Lefebvre).

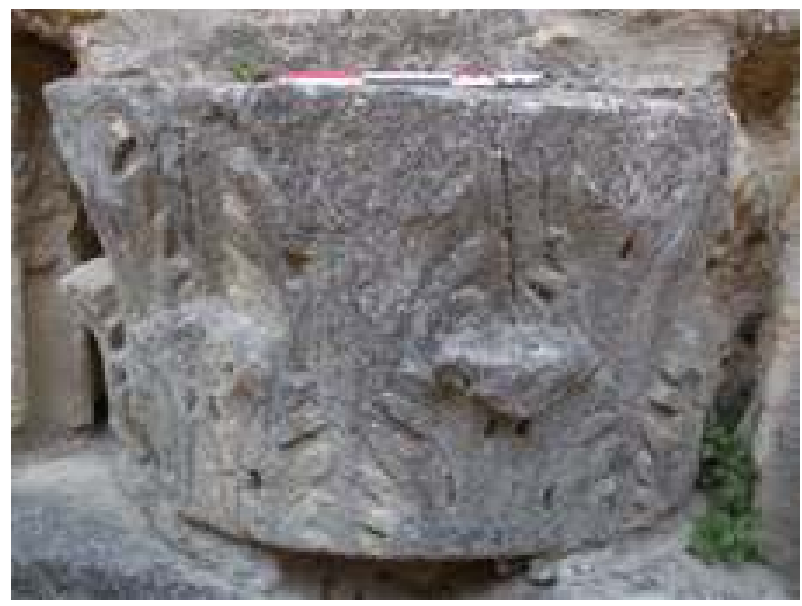

Fig. 18 - Moitié inférieure d'un chapiteau corinthien de colonne réemployé dans la fondation du chevet de la cathédrale NotreDame-de-Nazareth ( $n^{\circ}$ inv. : VAI.CATH.09) (C. Lefebvre).

La fondation du chevet ne constitue pas le seul lieu de la cathédrale où l'on trouve des réemplois. Présents dans son élévation externe, ils sont parfois retaillés en moellons tout en conservant leur décor. Il est aisé d'identifier des fragments d'inscription, de rinceaux, d'une colonne ou d'un pilastre cannelé, etc. (fig. 20). Il apparaît également que l'orne-

goutte créées au contact des digitations de deux lobes contigus d'une même feuille, de l'espace du calathos laissé vide entre les hélices et les volutes, et de ces dernières très proéminentes venant soutenir les cornes d'abaque.

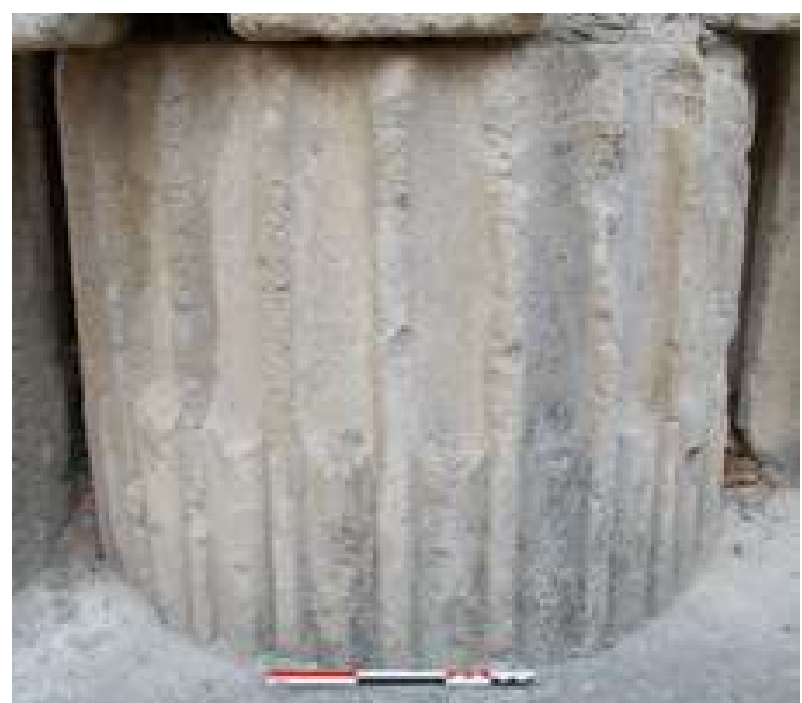

Fig. 17 - Tambour d'un fût cannelé de colonne orné de 24 cannelures partiellement rudentées et d'un diamètre de $90 \mathrm{~cm}$, réemployé dans la fondation du chevet de la cathédrale NotreDame-de-Nazareth ( $n^{\circ}$ inv. : VAI.CATH.14) (C. Lefebvre).

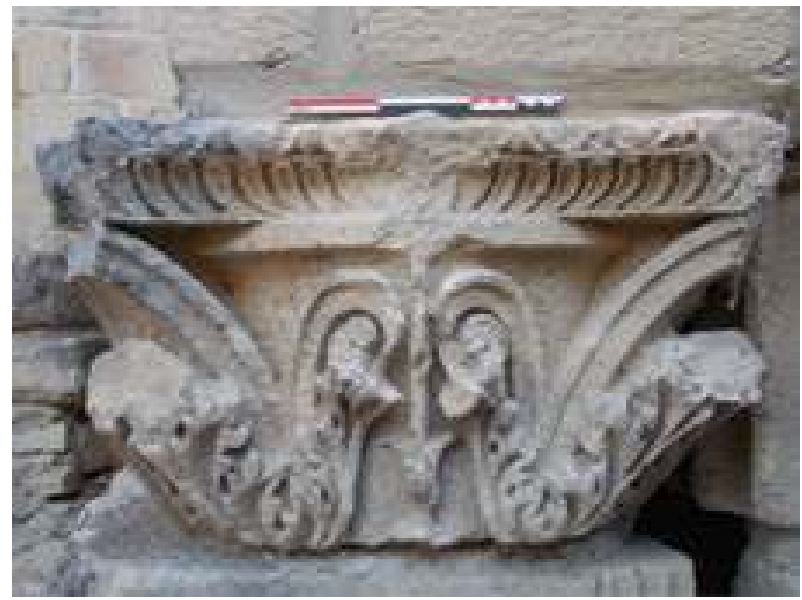

Fig. 19 - Moitié supérieure d'un chapiteau corinthien de colonne réemployé dans la fondation du chevet de la cathédrale NotreDame-de-Nazareth ( $\mathrm{n}^{\circ}$ inv. : VAI.CATH.06) (C. Lefebvre).

mentation sommitale de la cathédrale présente en deux endroits des blocs réemployés. Cette hypothèse, qui a pu être avancée au cours de la mission "Vaison 3D ", concerne la frise de rinceaux ornant le bas-côté méridional et la corniche couronnant la façade sud de la nef. À l'heure actuelle, les deux ensembles ont pu être observés depuis une nacelle et nécessitent assurément d'être étudiés plus minutieusement et d'être l'objet de relevés. Il est toutefois possible de relever à partir des clichés photographiques réalisés des divergences dans la typologie du décor et sa sculpture. 

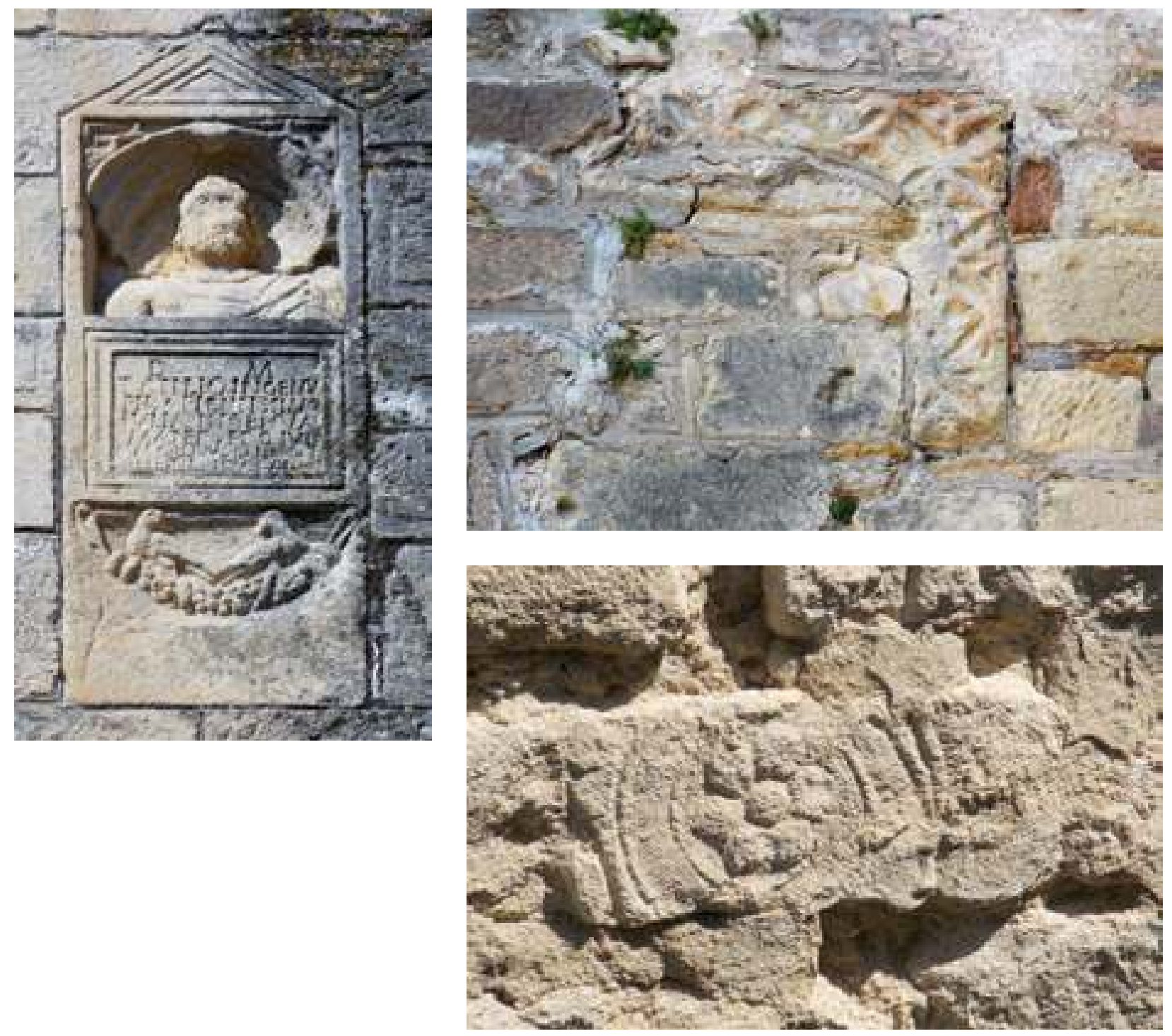

Fig. 20a-d - Réemplois divers dans les élévations extérieures de la cathédrale Notre-Dame-deNazareth conservés dans leur forme initiale ou retaillés en moellons (C. Lefebvre).

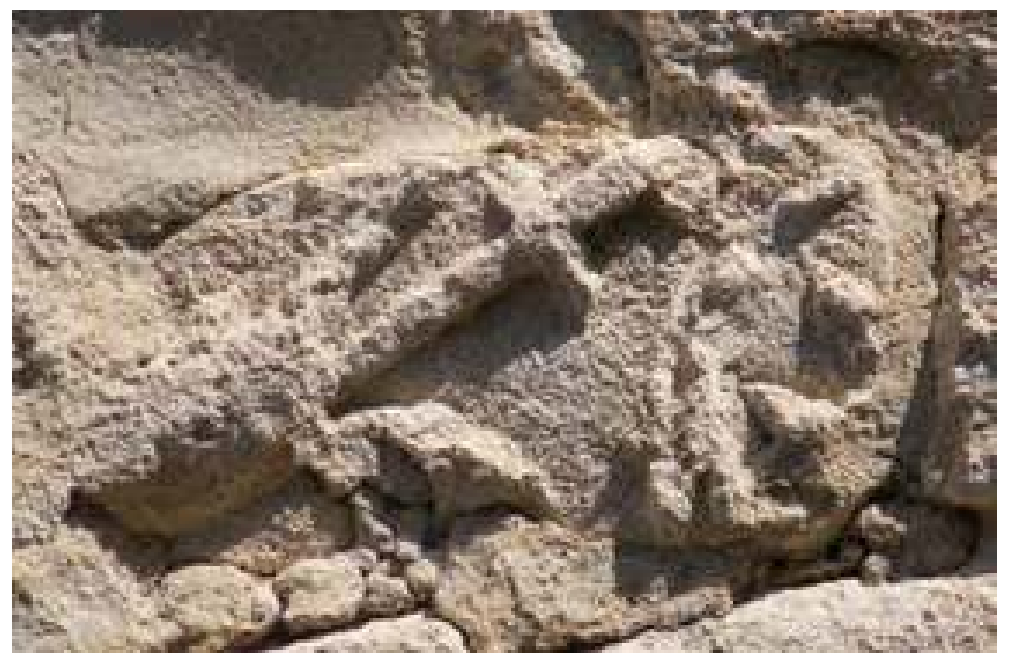



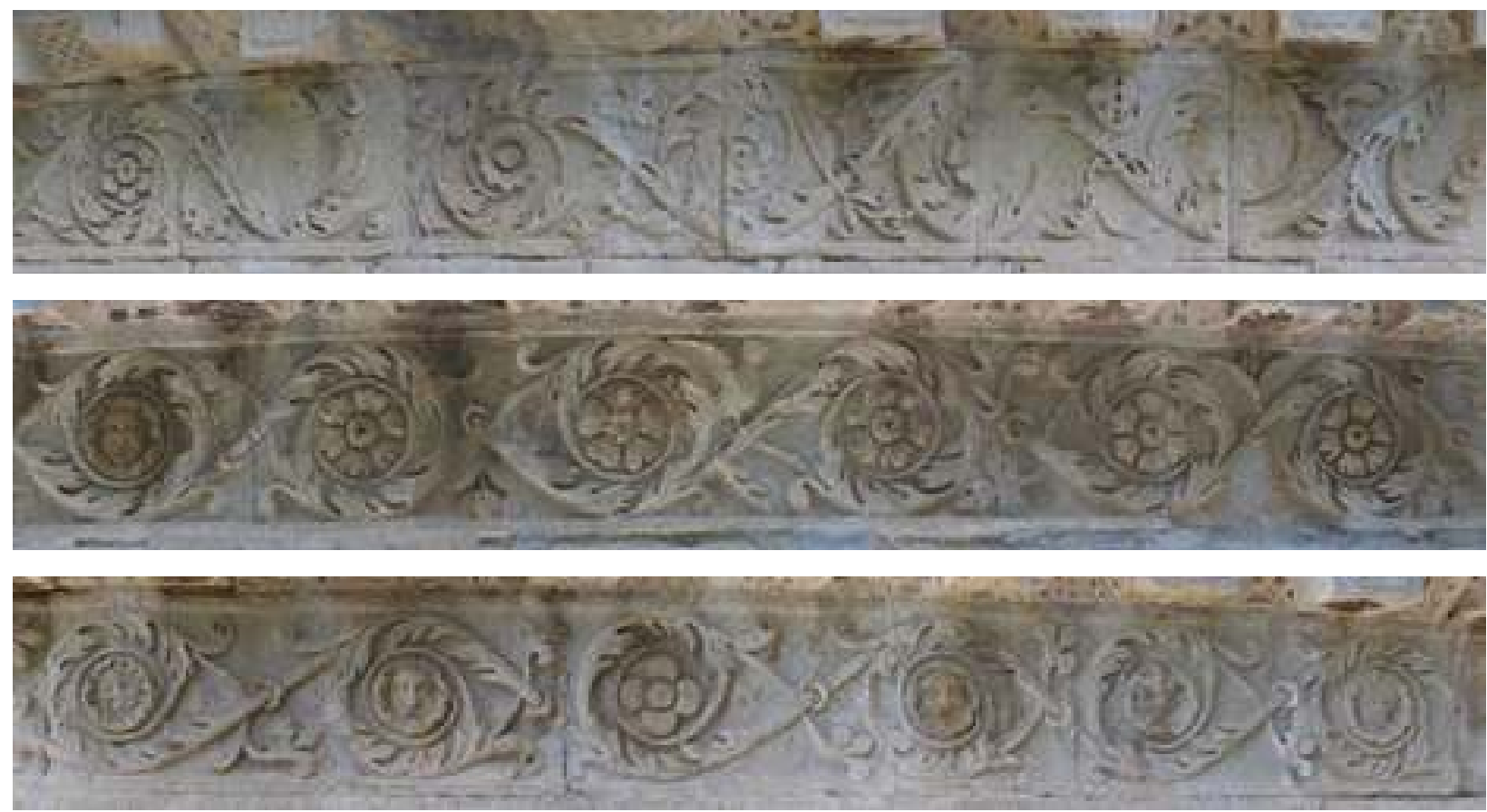

Fig. 21a-c - Détails de la frise qui orne le bas-côté méridional de la cathédrale Notre-Dame-de-Nazareth (détail de la frise entre le premier et le deuxième contrefort à compter de l'est ; détail de la frise entre le deuxième et le troisième contrefort; détail de la frise entre le troisième et le quatrième contrefort) (C. Lefebvre).

Dans la frise du bas-côté méridional (fig. 21), à l'extrémité orientale de la façade entre les premier et deuxième contreforts, les tiges des rinceaux sont finement sculptées comme les acanthes qui les recouvrent et s'enroulent pour laisser place à des fleurons. Les tiges donnent également naissance à divers motifs végétaux dans les écoinçons laissés libres par les rinceaux. Entre les deuxième et troisième contreforts, les rinceaux perdent de leur finesse, les tiges et les feuilles s'épaississent et les écoinçons sont bien moins décorés. Les fleurons présentent un traitement plus rigide et ils sont parfois remplacés par des visages humains. À l'ouest du troisième contrefort, cette sculpture plus grossière et plus pauvre en motifs végétalisés se poursuit jusqu'à l'extrémité occidentale de la façade. Il existe donc, entre le deuxième et le troisième contrefort, un changement stylistique dû à la présence de deux ou trois types de décors. Cette différence de traitement révèle la présence d'une

Fig. 22a-b - Détails d'est en ouest de la corniche qui orne la façade méridionale de la nef de la cathédrale Notre-Dame-de-Nazareth (détail de la corniche à l'angle sud-est de la façade méridionale; détail de la corniche au-dessus du premier contrefort) (C. Lefebvre).
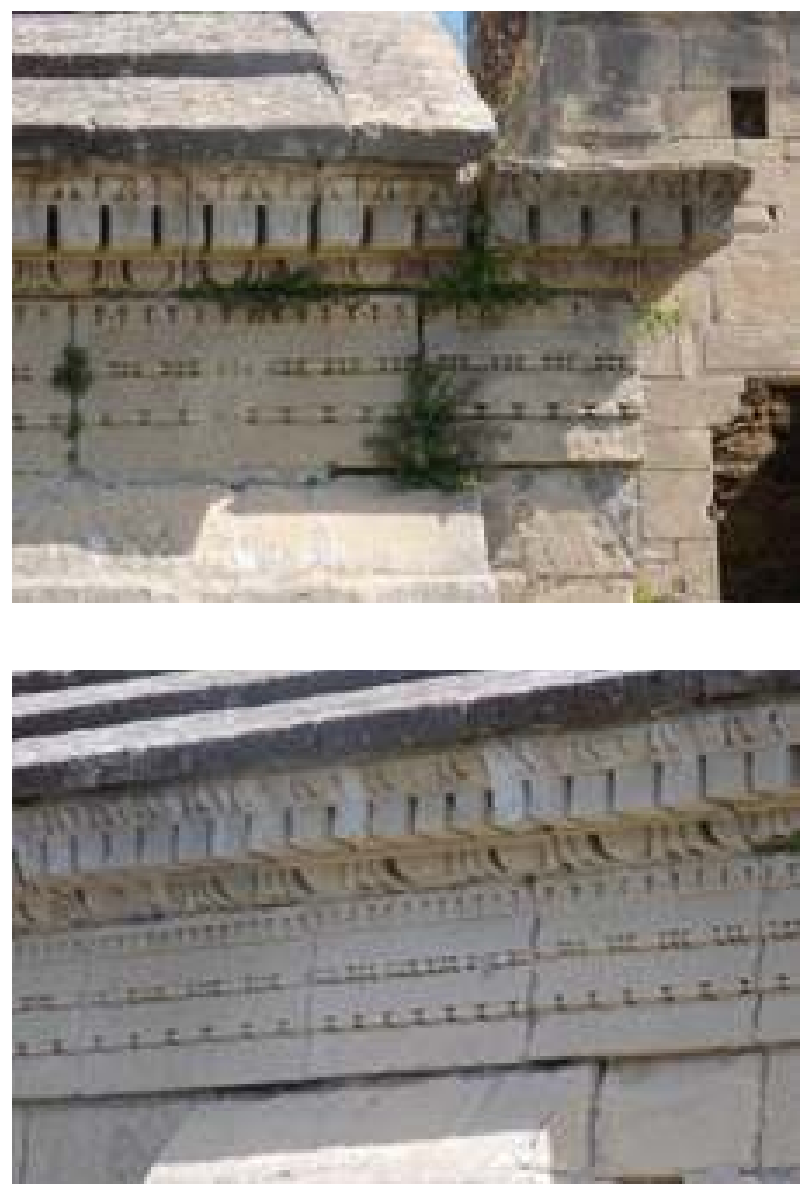


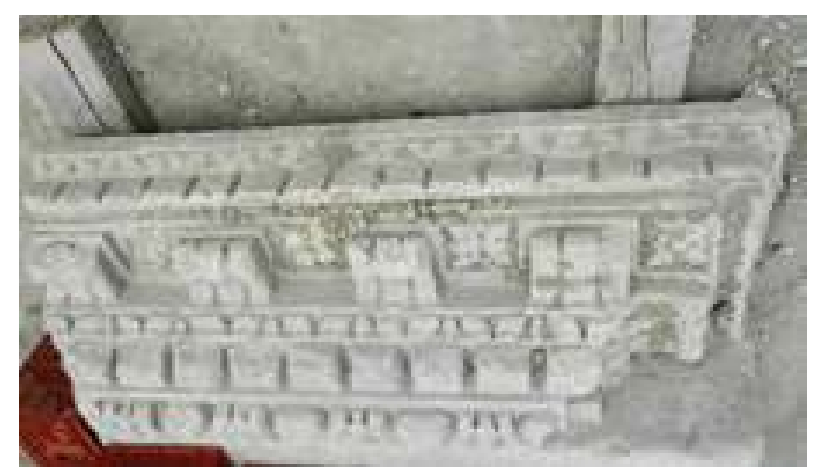

Fig. 23 - Face de parement extérieure d'un bloc de corniche du portique occidental du forum de Vaison-la-Romaine découvert en 2013 durant la première campagne de fouilles programmées sous la direction de J.-M. Mignon (C. Lefebvre).

sculpture antique et d'une sculpture médiévale et suggère le réemploi de frises à rinceaux d'époque romaine. Ce changement stylistique reste toutefois difficile à déterminer puisque l'on constate une véritable volonté des sculpteurs au Moyen-Âge à reproduire et respecter au mieux le modèle antique.

La question du réemploi se pose également pour la corniche de la façade sud de la nef (fig. 22). Deux traitements du décor ont été distingués entre les blocs qui occupent l'extrémité orientale de la façade et le restant de la corniche. Les premiers présentent des motifs ornementaux et une ordonnance qui se retrouve notamment sur le registre inférieur d'un bloc de corniche du portique occidental du forum (fig. 23). La découverte en 2011 de ce complexe architectural situé non loin de la cathédrale vers l'est (fig. 1) invite donc à se demander si certains blocs de corniche possiblement réemployés dans l'ornementation de la nef pourraient provenir du forum. Si tel était le cas, l'hypothèse pourrait aussi être envisagée pour d'autres éléments réemployés tels que ceux de la frise et la fondation du chevet.

L'analyse des réemplois de la cathédrale Notre-Dame-de-Nazareth suscite de nombreux questionnements et ouvre de nouvelles pistes de réflexion sur la construction même de l'édifice et les méthodes de réemploi qui furent pratiquées. Il apparaît assurément que pour la fondation du chevet, le réemploi d'éléments antiques résulte avant tout d'une raison pratique et économique avec des matériaux de dimensions considérables et aux formes avantageuses. Toutefois, devons-nous pour autant écarter l'éventualité d'une valeur symbolique qui ressort clairement avec les réemplois des blocs de frise et de corniche dans l'élévation. La réutilisation d'éléments issus d'édifices monumentaux romains en partie sommitale de l'édifice religieux et la mise en valeur de leur ornementation laissée volontairement apparente et reproduite avec soin n'exprime-t-elle pas une référence au passé et une volonté d'en conserver le souvenir? Cette notion symbolique probablement trop souvent écartée en faveur de l'économie ou de la facilité d'usage des réemplois semble pourtant avoir tout son intérêt dans une telle étude.

\section{CONCLUSION}

La ville de Vaison-la-Romaine ne peut être considérée comme un exemple exceptionnel de la pratique du réemploi architectural. Cependant, si une particularité mérite d'être soulignée ici, c'est très certainement la diversité des constructions faisant usage du réemploi, de leur fonction et de leur chronologie, qui s'étend du $\mathrm{I}^{\mathrm{er}}$ siècle de notre ère jusqu'au Moyen-Âge.

Adopté pour la construction du mur-digue de l'Ouvèze, aménagement public de la fin du I ${ }^{\text {er }}$ siècle, pour des constructions de l'Antiquité tardive dont l'identification reste incertaine ou encore pour la cathédrale Notre-Dame-de-Nazareth, édifice religieux des $\mathrm{XI}^{\mathrm{e}}$ et $\mathrm{XII}^{\mathrm{e}}$ siècles, le réemploi ne semble contraint ni par un espace-temps, ni par la fonction de la nouvelle construction à laquelle il est destiné.

À Vaison, le matériau des réemplois ne semble pas constituer un élément significatif du fait de l'utilisation massive du calcaire de Beaumont-duVentoux dans l'architecture publique de la ville romaine et donc de sa disponibilité.

La nature du réemploi semble donc apparaître comme le principal critère qui a été pris en compte pour le choix des blocs. Parfois utilisés dans le blocage des maçonneries, les pièces recyclées constituent des déchets fragmentaires sans valeur. Dans les fondations du chevet de la cathédrale, les blocs architecturaux n'étaient pas visibles mais l'intérêt pour leur forme et l'organisation structurée en rangée et en assise témoigne d'une logique de mise en œuvre. Celle-ci nécessita probablement le démantèlement d'un monument romain peut-être encore en élévation pour établir la fondation de l'édifice religieux et donc l'organisation d'un chantier de récupération avec une sélection en amont des 
éléments à prélever. Ces blocs ornés, au vu de leurs importantes dimensions, étaient difficiles à réemployer sans être retaillés ce qui impliquait un certain coût. De fait, ils étaient disposés dans les fondations ou bien ils retrouvaient leur fonction première, la cathédrale Notre-Dame-de-Nazareth nous offre les deux cas. Les valeurs pratiques et économiques de ces réemplois semblent donc évidentes. Cependant, ils invitent à s'interroger sur une valeur symbo- lique qui apparaît également dans la réutilisation des blocs de frise et de corniche qui couronnent au sud le bas-côté et la nef. Ici, le réemploi adopte un rôle ornemental reflétant un attachement au passé avec une volonté de l'exposer, de le reproduire et de le préserver. Cette mise en valeur du décor architectural trouve alors un intérêt particulier à l'époque médiévale avec une référence à l'antique très souvent reconnue dans l'architecture romane.

\section{Bibliographie}

Fellague 2007 = D. Fellague, L'architecture publique de Lugdunum. Les monuments et leur décor du I Ir s. av. J.-C. au $\mathrm{III}^{e}$ s. apr. J.-C., thèse de doctorat de l'Université Lumière Lyon II, sous la dir. de J.-Ch. Moretti, Lyon, 2007, p. 658-684.

Ginouvès - Martin 1985 = R. Ginouvès, R. Martin, Dictionnaire méthodique de l'architecture grecque et romaine. Tome I, matériaux, techniques de construction, techniques et formes du décor, Athènes, Rome, 1985.

Goudineau - de Kisch 1991 = Chr. Goudineau, Y. de Kisch, Vaison-la-Romaine, Paris, 1991, p. 9, 11 et 131.

Goudineau - de Kisch 1999 = Chr. Goudineau, Y. de Kisch, Vaison-la-Romaine, Guides archéologiques de la France, 1, Avignon, 1999, p. 5-6.

Gros $1979=$ P. Gros, Pour une chronologie des arcs de triomphe de Gaule Narbonnaise (à propos de l'arc de Glanum), dans Gallia, 37.1, 1979, p. 55-83.

Kauffmann 1979 = A. Kauffmann, Vaison-la-Romaine. Fouille du terrain situé au nord de la cathédrale. Rapport de fouille sur la campagne de juillet 1979, Aix-en-Provence, 1979.

Michel d'Annoville $1994=$ C. Michel d'Annoville, Vaison, la cathédrale, dans Bilan Scientifique 1993, 1994, p. 230231.

Mignon 1994 = J.-M. Mignon, Vaison, les rives de l'Ouvèze, dans Bilan Scientifique 1993, 1994, p. 231.

Mignon 1997 = J.-M. Mignon, Vaison, sur la rive gauche de l'Ouvèze, un mur-digue, dans Bilan Scientifique 1996, 1997, p. 168-170.
Provost - Meffre $2003=$ M. Provost, J.-Cl. Meffre, Vaison la-Romaine et ses campagnes, 84/1, Paris, 2003 (Carte Archéologique de la Gaule).

Salet 1951 = F. Salet, Découvertes à la cathédrale de Vaison, dans Bulletin Municipal, 109, 1951, p. 79-80.

Sautel 1926 = J. Sautel, Vaison dans l'Antiquité, vol. II : catalogue des objets romains trouvés à Vaison et dans son territoire, Avignon, 1926, p. 85-86.

Sautel $1948=\mathrm{J}$. Sautel, Informations archéologiques, $X I I I^{\bullet}$ Circonscription: Ardèche, Drôme, Vaucluse, dans Gallia, 6.1, 1948, p. 218-222.

Sautel 1950a = J. Sautel, Les origines de la cathédrale NotreDame-de-Nazareth à Vaison-la-Romaine, extrait du Bulletin des Facultés Catholiques, 1950.

Sautel $1950 \mathrm{~b}=$ J. Sautel, Bulletin archéologique. Fouilles et découvertes de la période historique, dans Mémoires de l'Académie de Vaucluse, 10, 1948-1949, p. 165-166.

Sautel 1950-1951 = J. Sautel, Les fouilles de l'abside de la cathédrale de Vaison, dans Bulletin de la société nationale des antiquaires de France, 1950-1951, p. 203-204.

Tardy 1989 = D. Tardy, Le décor architectonique de Saintes antiques, I: les chapiteaux et les bases, $5^{\mathrm{e}}$ suppl. Aquitania, Bordeaux, 1989.

Tardy 1994 = D. Tardy, Le décor architectonique de Saintes antique, II: les entablements, $7^{\mathrm{e}}$ suppl. Aquitania, Bordeaux, 1994.

Tardy 2005 = D. Tardy, Le décor architectonique de Vesunna (Périgueux antique), 12 $2^{\mathrm{e}}$ suppl. Aquitania, Pessac, 2005. 
